MARCH 1998, PAGES 1-35

\title{
INFLUENCE OF NATURAL CONVECTION ON STABILITY OF REACTION FRONTS IN LIQUIDS
}

\author{
BY \\ M. GARBEY, A. TAÏK, AND V. VOLPERT \\ Analyse Numérique, UMR 5585 CNRS, Université Lyon 1, France
}

\begin{abstract}
We study the influence of natural convection on stability of reaction fronts in liquids. In our previous article [6] we considered the case where the reactants were in a liquid phase and the product of the reaction was solid. In this paper we study the case where both of them are liquid. We carry out a linear stability analysis and show that the results are essentially different compared to the case of a solid product.
\end{abstract}

1. Introduction. There are different types of instabilities of reaction fronts. We mention thermo-diffusional instability, hydrodynamical instability, and convective instability as examples.

The thermo-diffusional instability appears as a result of competition between heat production in the reaction zone and heat transfer from the reaction zone to the cold original reactants. To study this type of instability, the density of the medium can be taken to be constant to remove the influence of hydrodynamics and to simplify the model. The stability conditions in this case were studied in [1], [2], [11], [14], and [22].

A hydrodynamical instability of the reaction front can occur if the density of the medium is variable. Usually it is considered as a given function of the temperature. The instability is caused by heat expansion of the gas or liquid in the neighbourhood of the reaction zone, [3], [7], [8], [13], and [28].

By convective instability, we mean that which appears due to natural convection. This instability can be studied in the pure form if we consider the Boussinesq approximation, i.e., we neglect the change of density everywhere except for the buoyancy term. The Boussinesq approximation for the gas combustion was justified and used to study the front's stability in [14], [16].

Different types of instabilities can interact. It is known that for gas combustion the natural convection influences the conditions for a hydrodynamical instability [28]. Recent experiments [19] show an interaction between the hydrodynamical and thermodiffusional instability for polymerization fronts. Intensity of a turbulent liquid motion near the reaction front caused by the hydrodynamical instability is not constant. It

Received December 1, 1994.

1991 Mathematics Subject Classification. Primary 76E15, 80A32.

(C)1998 Brown University 
depends on time periodically, according to the one-dimensional pulsations that appear as a result of the thermo-diffusional instability. Some multidimensional modes where the liquid motion forms a spatial structure that changes periodically in time also seem to be observed. However, they are seen less clearly than one-dimensional pulsations.

The theoretical analysis of a one-dimensional model [26] shows that the heat expansion of the liquid makes the front less stable. This is in accordance with the experimental results where one-dimensional pulsations are observed below the limit predicted by the theory for reaction fronts in solids.

The influence of natural convection on the stability of reaction fronts in liquids was studied in [6]. This work was devoted to the case where the product of the reaction was solid. An example of reaction fronts with liquid reactants and solid products is provided by frontal polymerization, [6], [19]. We have shown that the front propagating upward can cause a cellular instability; for the front propagating downward it cannot take place. This is to some extent similar to the Rayleigh-Bénard convection in the layers of the liquid heated from below or above.

The natural convection also changes the conditions of the oscillatory thermo-diffusional instability. Moreover, this influence depends on the direction of the front propagation. We show that the front propagating upward becomes more stable than without the hydrodynamics, while the front propagating downward is less stable. This explains why the thermo-diffusional instability was observed experimentally only for descending fronts and for values of the parameters where the reaction front in a solid should be stable.

In this work we study stability of reaction fronts in the case where also the product of the reaction is liquid. This situation can occur for frontal polymerization as well as for some other processes, [20]-[21], [23]-[24], [25], [27]. The results of the analysis appear to be essentially different with respect to the case of a solid product.

2. The mathematical model. The mathematical model used here is close to that in the case of a solid product [6]. However, in the case of a liquid product we should also consider the equations of motion behind the reaction zone. As will be clear later, this changes the jump conditions in the reaction zone and can influence the stability conditions in an essential way.

We consider the heat equation, the equation for the concentration, and the NavierStokes equations under the Boussinesq approximation:

$$
\begin{gathered}
\frac{\partial T}{\partial t}+v \nabla T=\kappa \Delta T+q W, \\
\frac{\partial \alpha}{\partial t}+v \nabla \alpha=W, \\
\frac{\partial v}{\partial t}+(v \nabla) v=-\frac{1}{\rho} \nabla p+\nu \Delta v+g \beta\left(T-T_{0}\right) \gamma, \\
\operatorname{div} v=0,
\end{gathered}
$$

subject to the boundary conditions:

$$
\text { as } z \rightarrow+\infty, \quad T=T_{i}, \alpha=0, \text { and } v=0,
$$


and

$$
\text { as } z \rightarrow-\infty, \quad T=T_{b}, \alpha=1 \text {, and } v=0 \text {. }
$$

Here

$$
\nabla=\left(\frac{\partial}{\partial x}, \frac{\partial}{\partial y}, \frac{\partial}{\partial z}\right), \quad \Delta=\frac{\partial^{2}}{\partial x^{2}}+\frac{\partial^{2}}{\partial y^{2}}+\frac{\partial^{2}}{\partial z^{2}},
$$

$x, y, z$ are the spatial variables, $-\infty<x, y, z<+\infty, T$ is the temperature, $\alpha$ is the concentration of the reaction product, $v$ is the velocity of the medium, $p$ is the pressure, $\kappa$ is the coefficient of thermal diffusivity, $q$ is the adiabatic heat release of the reaction, $\rho$ is the density, $\nu$ is the viscosity, $\gamma$ is the unit vector in the vertical direction (upward), $\beta$ is the coefficient of thermal expansion, $g$ is the gravity acceleration, $T_{0}$ is an average value of the temperature, $T_{i}$ is the initial temperature, and $T_{b}$ is the temperature of the burned mixture given by $T_{b}=T_{i}+q$.

This model is formulated under the following assumptions:

The chemical reaction is a one-step reaction of order zero. The reaction rate is of the form

$$
W=k(T) \phi(\alpha)
$$

where

$$
\phi(\alpha)= \begin{cases}1 & \text { if } \alpha<1 \\ 0 & \text { if } \alpha=1\end{cases}
$$

The temperature dependence of the reaction rate is given by the Arrhenius law

$$
k(T)=k_{0} \exp \left(-E /\left(R_{0} T\right)\right),
$$

where $k_{0}$ is the pre-exponential factor, $E$ is the activation energy (presumed to be large), and $R_{0}$ is the universal gas constant, the liquid is incompressible, and in the equation for the concentration we neglect the diffusion term since the diffusion coefficient for liquids is usually much less than that of thermal diffusivity.

The equations are nondimensionalized by introducing the dimensionless variables:

$$
\begin{gathered}
x_{1}=\frac{x c_{1}}{\kappa}, \quad y_{1}=\frac{y c_{1}}{\kappa}, \quad z_{1}=\frac{z c_{1}}{\kappa}, \quad t_{1}=\frac{t c_{1}^{2}}{\kappa}, \quad p_{1}=\frac{p}{c_{1}^{2} \rho}, \quad c_{1}=\frac{c}{\sqrt{2}}, \\
\theta=\frac{T-T_{b}}{q} \text { and } \quad c^{2}=\frac{2 k_{0} \kappa}{2} \frac{R_{0} T_{b}^{2}}{E} \exp \left(-E /\left(R_{0} T_{b}\right)\right) .
\end{gathered}
$$

Here $c$ gives the velocity of the stationary front in the limit of large Zeldovich number [18]. For convenience we keep the same notation for the spatial coordinates and the pressure. The system $(2.1)-(2.4)$ becomes

$$
\begin{gathered}
\frac{\partial \theta}{\partial t}+v \nabla \theta=\Delta \theta+Z \exp \left(\theta /\left(Z^{-1}+\delta \theta\right)\right) \phi(\alpha), \\
\frac{\partial \alpha}{\partial t}+v \nabla \alpha=Z \exp \left(\theta /\left(Z^{-1}+\delta \theta\right)\right) \phi(\alpha), \\
\frac{\partial v}{\partial t}+(v \nabla) v=-\nabla p+P \Delta v+P R\left(\theta+\theta^{0}\right) \gamma, \\
\operatorname{div} v=0,
\end{gathered}
$$


with the boundary conditions:

$$
\begin{gathered}
\text { as } z \rightarrow+\infty, \quad \theta=-1, \alpha=0, \text { and } v=0, \\
\text { as } z \rightarrow-\infty, \quad \theta=0, \alpha=1, \text { and } v=0 .
\end{gathered}
$$

Here $P=\nu / \kappa$ is the Prandtl number, $R=\left(g \beta q \kappa^{2}\right) /\left(\nu c^{3}\right)$ is the Rayleigh number, $Z=q E /\left(R_{0} T_{b}^{2}\right)$ is the Zeldovich number, $\delta=R_{0} T_{b} / E$, and $\theta^{0}=\left(T_{b}-T_{0}\right) / q$.

3. The asymptotic derivation of the model. To study the problem analytically we use a conventional approach for combustion problems based on matching asymptotic expansions (see [5], [6], [14], [15], [28], [29]). It allows us to reduce the problem (2.5)-(2.8) to an interface problem.

We carry out the formal asymptotic analysis with $\varepsilon=Z^{-1}$ as a small parameter, and derive a closed interface problem.

Let $\zeta(x, y, t)$ denote the location of the reaction zone and introduce the new independent variable:

$$
z_{1}=z-\zeta(x, y, t)
$$

Then we rewrite $(2.5)-(2.8)$ in the form

$$
\begin{gathered}
\frac{\partial \theta}{\partial t}-\frac{\partial \theta}{\partial z_{1}} \frac{\partial \zeta}{\partial t}+v \tilde{\nabla} \theta=\tilde{\Delta} \theta+Z \exp \left(\theta /\left(Z^{-1}+\delta \theta\right)\right) \phi(\alpha) \\
\frac{\partial \alpha}{\partial t}-\frac{\partial \alpha}{\partial z_{1}} \frac{\partial \zeta}{\partial t}+v \tilde{\nabla} \alpha=Z \exp \left(\theta /\left(Z^{-1}+\delta \theta\right)\right) \phi(\alpha) \\
\frac{\partial v}{\partial t}-\frac{\partial v}{\partial z_{1}} \frac{\partial \zeta}{\partial t}+(v \tilde{\nabla}) v=-\tilde{\nabla} p+P \tilde{\Delta} v+Q\left(\theta+\theta^{0}\right) \gamma \\
\frac{\partial v_{x}}{\partial x}-\frac{\partial v_{x}}{\partial z_{1}} \frac{\partial \zeta}{\partial x}+\frac{\partial v_{y}}{\partial y}-\frac{\partial v_{y}}{\partial z_{1}} \frac{\partial \zeta}{\partial y}+\frac{\partial v_{z}}{\partial z_{1}}=0
\end{gathered}
$$

where

$$
\begin{gathered}
\tilde{\Delta}=\frac{\partial^{2}}{\partial x^{2}}+\frac{\partial^{2}}{\partial y^{2}}+\frac{\partial^{2}}{\partial z_{1}^{2}}-2 \frac{\partial^{2}}{\partial x \partial z_{1}} \frac{\partial \zeta}{\partial x}-2 \frac{\partial^{2}}{\partial y \partial z_{1}} \frac{\partial \zeta}{\partial y} \\
+\frac{\partial^{2}}{\partial z_{1}^{2}}\left(\left(\frac{\partial \zeta}{\partial x}\right)^{2}+\left(\frac{\partial \zeta}{\partial y}\right)^{2}\right)-\frac{\partial}{\partial z_{1}}\left(\frac{\partial^{2} \zeta}{\partial x^{2}}+\frac{\partial^{2} \zeta}{\partial y^{2}}\right) \\
\tilde{\nabla}=\left(\frac{\partial}{\partial x}-\frac{\partial}{\partial z_{1}} \frac{\partial \zeta}{\partial x}, \frac{\partial}{\partial y}-\frac{\partial}{\partial z_{1}} \frac{\partial \zeta}{\partial y}, \frac{\partial}{\partial z_{1}}\right)
\end{gathered}
$$

and

$$
Q=P R \text {. }
$$

We seek the outer expansion of problem (3.1)-(3.4) in the form

$$
\begin{array}{llrl}
\theta & =\theta_{0}+\varepsilon \theta_{1}+\cdots, & & \alpha=\alpha_{0}+\varepsilon \alpha_{1}+\cdots, \\
v & =v_{0}+\varepsilon v_{1}+\cdots, & & p=p_{0}+\varepsilon p_{1}+\cdots .
\end{array}
$$

To obtain the jump conditions in the reaction zone we consider the inner problem using the stretching coordinate $\eta$ defined by $\eta=z_{1} \varepsilon^{-1}$. 
We look for the inner solution in the form

$$
\begin{gathered}
\theta=\varepsilon \tilde{\theta}_{1}+\cdots, \quad \alpha=\tilde{\alpha}_{0}+\varepsilon \tilde{\alpha}_{1}+\cdots \\
v=\tilde{v}_{0}+\varepsilon \tilde{v}_{1}+\cdots, \quad p=\tilde{p}_{0}+\varepsilon \tilde{p}_{1}+\cdots, \quad \zeta=\zeta_{0}+\varepsilon \zeta_{1}+\cdots
\end{gathered}
$$

Substituting (3.5)-(3.6) into (3.1)-(3.4), equating coefficients of like powers of $\varepsilon$, and limiting ourselves to $O\left(\varepsilon^{2}\right)$, we obtain:

Order $\varepsilon^{-2}$ :

$$
P\left(1+\left(\frac{\partial \zeta_{0}}{\partial x}\right)^{2}+\left(\frac{\partial \zeta_{0}}{\partial y}\right)^{2}\right) \frac{\partial^{2} \tilde{v}_{0}}{\partial \eta^{2}}=0
$$

Order $\varepsilon^{-1}$ :

$$
\begin{gathered}
\left(1+\left(\frac{\partial \zeta_{0}}{\partial x}\right)^{2}+\left(\frac{\partial \zeta_{0}}{\partial y}\right)^{2}\right) \frac{\partial^{2} \tilde{\theta}_{1}}{\partial \eta^{2}}+\exp \left(\tilde{\theta}_{1} /\left(1+\delta \tilde{\theta}_{1}\right)\right) \phi\left(\tilde{\alpha}_{0}\right)=0 \\
-\frac{\partial \tilde{\alpha}_{0}}{\partial \eta} \frac{\partial \zeta_{0}}{\partial t}-\frac{\partial \tilde{\alpha}_{0}}{\partial \eta}\left(\tilde{v}_{0_{x}} \frac{\partial \zeta_{0}}{\partial x}+\tilde{v}_{0_{y}} \frac{\partial \zeta_{0}}{\partial y}-\tilde{v}_{0_{z}}\right)=\exp \left(\tilde{\theta}_{1} /\left(1+\delta \tilde{\theta}_{1}\right)\right) \phi\left(\tilde{\alpha}_{0}\right) \\
-\frac{\partial \tilde{v}_{0_{x}}}{\partial \eta} \frac{\partial \zeta_{0}}{\partial t}-\tilde{v}_{0_{x}} \frac{\partial \tilde{v}_{0}}{\partial \eta} \frac{\partial \zeta_{0}}{\partial x}-\tilde{v}_{0_{y}} \frac{\partial \tilde{v}_{0}}{\partial \eta} \frac{\partial \zeta_{0}}{\partial y}+\tilde{v}_{0_{z}} \frac{\partial \tilde{v}_{0}}{\partial \eta}=t_{0} \frac{\partial \tilde{p}_{0}}{\partial \eta}+P\left(A \frac{\partial^{2} \tilde{v}_{1}}{\partial \eta^{2}}+F_{0} \frac{\partial \tilde{v}_{0}}{\partial \eta}\right) \\
-\frac{\partial \tilde{v}_{0_{x}}}{\partial \eta} \frac{\partial \zeta_{0}}{\partial x}-\frac{\partial \tilde{v}_{0_{y}}}{\partial \eta} \frac{\partial \zeta_{0}}{\partial y}+\frac{\partial \tilde{v}_{0_{z}}}{\partial \eta}=0
\end{gathered}
$$

Order $\varepsilon^{0}$ :

$$
\begin{aligned}
\frac{\partial \tilde{v}_{0}}{\partial t}- & \frac{\partial \tilde{v}_{1}}{\partial \eta} \frac{\partial \zeta_{0}}{\partial t}-\frac{\partial \tilde{v}_{0}}{\partial \eta} \frac{\partial \zeta_{1}}{\partial t}+\tilde{v}_{0_{x}}\left(\frac{\partial \tilde{v}_{0}}{\partial x}-\frac{\partial \tilde{v}_{1}}{\partial \eta} \frac{\partial \zeta_{0}}{\partial x}-\frac{\partial \tilde{v}_{0}}{\partial \eta} \frac{\partial \zeta_{1}}{\partial x}\right)+\tilde{v}_{1_{x}} \frac{\partial \tilde{v}_{0}}{\partial \eta} \frac{\partial \zeta_{0}}{\partial x} \\
& +\tilde{v}_{1_{y}} \frac{\partial \tilde{v}_{0}}{\partial \eta} \frac{\partial \zeta_{0}}{\partial y}+\tilde{v}_{0_{y}}\left(\frac{\partial \tilde{v}_{0}}{\partial y}-\frac{\partial \tilde{v}_{1}}{\partial \eta} \frac{\partial \zeta_{0}}{\partial y}-\frac{\partial \tilde{v}_{0}}{\partial \eta} \frac{\partial \zeta_{1}}{\partial y}\right)+\tilde{v}_{0_{z}} \frac{\partial \tilde{v}_{1}}{\partial \eta}+\tilde{v}_{1_{z}} \frac{\partial \tilde{v}_{0}}{\partial \eta} \\
= & -\nabla_{0} \tilde{p}_{0}+t_{1} \frac{\partial \tilde{p}_{0}}{\partial \eta}+t_{0} \frac{\partial \tilde{p}_{1}}{\partial \eta} \\
& +P\left(A \frac{\partial^{2} \tilde{v}_{2}}{\partial \eta^{2}}+t_{3} \frac{\partial^{2} \tilde{v}_{1}}{\partial \eta^{2}}+F_{0} \frac{\partial \tilde{v}_{1}}{\partial \eta}+t_{4} \frac{\partial^{2} \tilde{v}_{0}}{\partial \eta^{2}}+F_{1} \frac{\partial \tilde{v}_{0}}{\partial \eta}+\Delta_{1} \tilde{v}_{0}\right)+Q \gamma \theta^{0}
\end{aligned}
$$

$$
\frac{\partial \tilde{v}_{0_{x}}}{\partial x}-\frac{\partial \tilde{v}_{1_{x}}}{\partial \eta} \frac{\partial \zeta_{0}}{\partial x}-\frac{\partial \tilde{v}_{0_{x}}}{\partial \eta} \frac{\partial \zeta_{1}}{\partial x}+\frac{\partial \tilde{v}_{0_{y}}}{\partial y}-\frac{\partial \tilde{v}_{1_{y}}}{\partial \eta} \frac{\partial \zeta_{0}}{\partial y}-\frac{\partial \tilde{v}_{0_{y}}}{\partial \eta} \frac{\partial \zeta_{1}}{\partial y}+\frac{\partial \tilde{v}_{1_{z}}}{\partial \eta}=0
$$


Order $\varepsilon^{1}$ :

$$
\begin{aligned}
& \frac{\partial \tilde{v}_{1}}{\partial t}-\left(\frac{\partial \tilde{v}_{2}}{\partial \eta} \frac{\partial \zeta_{0}}{\partial t}+\frac{\partial \tilde{v}_{1}}{\partial \eta} \frac{\partial \zeta_{1}}{\partial t}+\frac{\partial \tilde{v}_{0}}{\partial \eta} \frac{\partial \zeta_{2}}{\partial t}\right)+\tilde{v}_{0_{x}}\left(\frac{\partial \tilde{v}_{1}}{\partial x}-\frac{\partial \tilde{v}_{1}}{\partial \eta} \frac{\partial \zeta_{1}}{\partial x}-\frac{\partial \tilde{v}_{0}}{\partial \eta} \frac{\partial \zeta_{2}}{\partial x}-\frac{\partial \tilde{v}_{2}}{\partial \eta} \frac{\partial \zeta_{0}}{\partial x}\right) \\
&+ \tilde{v}_{1_{x}}\left(\frac{\partial \tilde{v}_{0}}{\partial x}-\frac{\partial \tilde{v}_{0}}{\partial \eta} \frac{\partial \zeta_{1}}{\partial x}-\frac{\partial \tilde{v}_{1}}{\partial \eta} \frac{\partial \zeta_{0}}{\partial x}\right)-\tilde{v}_{2_{x}} \frac{\partial \tilde{v}_{0}}{\partial \eta} \frac{\partial \zeta_{0}}{\partial x}+\tilde{v}_{0_{y}}\left(\frac{\partial \tilde{v}_{1}}{\partial y}-\frac{\partial \tilde{v}_{1}}{\partial \eta} \frac{\partial \zeta_{1}}{\partial y}-\frac{\partial \tilde{v}_{2}}{\partial \eta} \frac{\partial \zeta_{0}}{\partial y}\right) \\
&+\tilde{v}_{1_{y}}\left(\frac{\partial \tilde{v}_{0}}{\partial y}-\frac{\partial \tilde{v}_{0}}{\partial \eta} \frac{\partial \zeta_{1}}{\partial y}-\frac{\partial \tilde{v}_{1}}{\partial \eta} \frac{\partial \zeta_{0}}{\partial y}\right)-\tilde{v}_{2_{y}} \frac{\partial \tilde{v}_{0}}{\partial \eta} \frac{\partial \zeta_{0}}{\partial y}+\tilde{v}_{0_{z}} \frac{\partial \tilde{v}_{2}}{\partial \eta}+\tilde{v}_{1_{z}} \frac{\partial \tilde{v}_{1}}{\partial \eta}+\tilde{v}_{2_{z}} \frac{\partial \tilde{v}_{0}}{\partial \eta} \\
&= t_{0} \frac{\partial \tilde{p}_{2}}{\partial \eta}+t_{1} \frac{\partial \tilde{p}_{1}}{\partial \eta}-\nabla_{0} \tilde{p}_{1}+t_{2} \frac{\partial \tilde{p}_{0}}{\partial \eta}+P\left(A \frac{\partial^{2} \tilde{v}_{3}}{\partial \eta^{2}}+t_{3} \frac{\partial^{2} \tilde{v}_{2}}{\partial \eta^{2}}+F_{0} \frac{\partial \tilde{v}_{2}}{\partial \eta}\right. \\
&\left.+F_{1} \frac{\partial \tilde{v}_{1}}{\partial \eta}+\Delta_{1} \tilde{v}_{1}+F_{2} \frac{\partial \tilde{v}_{0}}{\partial \eta}\right)+Q \gamma \theta_{1},
\end{aligned}
$$

$$
\begin{aligned}
\frac{\partial \tilde{v}_{1_{x}}}{\partial x}-\frac{\partial \tilde{v}_{2_{x}}}{\partial \eta} \frac{\partial \zeta_{0}}{\partial x}-\frac{\partial \tilde{v}_{1_{x}}}{\partial \eta} \frac{\partial \zeta_{1}}{\partial x} & -\frac{\partial \tilde{v}_{0_{x}}}{\partial \eta} \frac{\partial \zeta_{2}}{\partial x}+\frac{\partial \tilde{v}_{1_{y}}}{\partial y}-\frac{\partial \tilde{v}_{2_{y}}}{\partial \eta} \frac{\partial \zeta_{0}}{\partial y} \\
& -\frac{\partial \tilde{v}_{1_{y}}}{\partial \eta} \frac{\partial \zeta_{1}}{\partial y}-\frac{\partial \tilde{v}_{0_{y}}}{\partial \eta} \frac{\partial \zeta_{2}}{\partial y}+\frac{\partial \tilde{v}_{2_{z}}}{\partial \eta}=0
\end{aligned}
$$

Order $\varepsilon^{2}$ :

$$
\begin{aligned}
\frac{\partial \tilde{v}_{2_{x}}}{\partial x}-\frac{\partial \tilde{v}_{3_{x}}}{\partial \eta} \frac{\partial \zeta_{0}}{\partial x} & -\frac{\partial \tilde{v}_{2_{x}}}{\partial \eta} \frac{\partial \zeta_{1}}{\partial x}-\frac{\partial \tilde{v}_{1_{x}}}{\partial \eta} \frac{\partial \zeta_{2}}{\partial x}-\frac{\partial \tilde{v}_{0_{x}}}{\partial \eta} \frac{\partial \zeta_{3}}{\partial x}+\frac{\partial \tilde{v}_{2_{y}}}{\partial y} \\
& -\frac{\partial \tilde{v}_{3_{y}}}{\partial \eta} \frac{\partial \zeta_{0}}{\partial y}-\frac{\partial \tilde{v}_{2_{y}}}{\partial \eta} \frac{\partial \zeta_{1}}{\partial y}-\frac{\partial \tilde{v}_{1_{y}}}{\partial \eta} \frac{\partial \zeta_{2}}{\partial y}-\frac{\partial \tilde{v}_{0_{y}}}{\partial \eta} \frac{\partial \zeta_{3}}{\partial y}+\frac{\partial \tilde{v}_{3_{z}}}{\partial \eta}=0 .
\end{aligned}
$$

Here

$$
\begin{gathered}
\Delta_{1}=\frac{\partial^{2}}{\partial x^{2}}+\frac{\partial^{2}}{\partial y^{2}}, \quad \nabla_{0}=\left(\frac{\partial}{\partial x}, \frac{\partial}{\partial y}, 0\right), \\
F_{i}=-2\left(\frac{\partial \zeta_{i}}{\partial x} \frac{\partial}{\partial x}+\frac{\partial \zeta_{i}}{\partial y} \frac{\partial}{\partial y}\right)-\left(\frac{\partial^{2} \zeta_{i}}{\partial x^{2}}+\frac{\partial^{2} \zeta_{i}}{\partial y^{2}}\right) I, \quad i=0,1,2, \\
t_{0}=\left(\frac{\partial \zeta_{0}}{\partial x}, \frac{\partial \zeta_{0}}{\partial y},-1\right), \quad t_{i}=\left(\frac{\partial \zeta_{i}}{\partial x}, \frac{\partial \zeta_{i}}{\partial y}, 0\right) \text { for } i=1,2, \quad t_{3}=2\left(\frac{\partial \zeta_{0}}{\partial x} \frac{\partial \zeta_{1}}{\partial x}+\frac{\partial \zeta_{0}}{\partial y} \frac{\partial \zeta_{1}}{\partial y}\right), \\
t_{4}=\left(\frac{\partial \zeta_{1}}{\partial x}\right)^{2}+\left(\frac{\partial \zeta_{1}}{\partial y}\right)^{2}+\frac{\partial \zeta_{0}}{\partial x} \frac{\partial \zeta_{2}}{\partial x}+\frac{\partial \zeta_{0}}{\partial y} \frac{\partial \zeta_{2}}{\partial y} \quad \text { and } \quad A=\left(1+\left(\frac{\partial \zeta_{0}}{\partial x}\right)^{2}+\left(\frac{\partial \zeta_{0}}{\partial y}\right)^{2}\right),
\end{gathered}
$$

where $I$ is the identity operator. The matching conditions as $\eta \rightarrow \pm \infty$ are (cf. Appendix A):

$$
\left.\tilde{v}_{0} \sim v_{0}\right|_{z_{1}= \pm 0}
$$




$$
\begin{gathered}
\tilde{v}_{1} \sim\left(\left.\frac{\partial v_{0}}{\partial z_{1}}\right|_{z_{1}= \pm 0}\right) \eta+\left.v_{1}\right|_{z_{1}= \pm 0} \\
\tilde{v}_{2} \sim \frac{1}{2}\left(\left.\frac{\partial^{2} v_{0}}{\partial z_{1}^{2}}\right|_{z_{1}= \pm 0}\right) \eta^{2}+\left(\left.\frac{\partial v_{1}}{\partial z_{1}}\right|_{z_{1}= \pm 0}\right) \eta+\left.v_{2}\right|_{z_{1}= \pm 0}
\end{gathered}
$$

and

$$
\tilde{v}_{3} \sim \frac{1}{6}\left(\left.\frac{\partial^{3} v_{0}}{\partial z_{1}^{3}}\right|_{z_{1}= \pm 0}\right) \eta^{3}+\frac{1}{2}\left(\left.\frac{\partial^{2} v_{1}}{\partial z_{1}^{2}}\right|_{z_{1}= \pm 0}\right) \eta^{2}+\left(\left.\frac{\partial v_{2}}{\partial z_{1}}\right|_{z_{1}= \pm 0}\right) \eta+\left.v_{3}\right|_{z_{1}= \pm 0}
$$

while as $\eta \rightarrow+\infty$ :

$$
\left.\tilde{\theta}_{1} \sim \theta_{1}\right|_{z_{1}=+0}+\left(\left.\frac{\partial \theta_{0}}{\partial z_{1}}\right|_{z_{1}=+0}\right) \eta, \quad \tilde{\alpha}_{0} \rightarrow 0
$$

and as $\eta \rightarrow-\infty$ :

$$
\left.\tilde{\theta}_{1} \sim \theta_{1}\right|_{z_{1}=-0}, \quad \tilde{\alpha}_{0} \rightarrow 1
$$

From (3.7), we obtain

$$
\frac{\partial^{2} \tilde{v}_{0}}{\partial \eta^{2}}=0
$$

Thus $\tilde{v}_{0}(\eta)$ is a linear function of $\eta$ and from the boundedness of the velocity it is identically constant. Using (3.17) we have

$$
\begin{gathered}
\left.v_{0}\right|_{z_{1}=+0}=\left.v_{0}\right|_{z_{1}=-0}, \\
\frac{\partial \tilde{v}_{0}}{\partial \eta}=0
\end{gathered}
$$

Thus the first term of the outer expansion of the velocity, $v_{0}$, is continuous at the front.

In view of (3.24), Eqs. (3.10), (3.13) take the form

$$
P A \frac{\partial^{2} \tilde{v}_{1}}{\partial \eta^{2}}+t_{0} \frac{\partial \tilde{p}_{0}}{\partial \eta}=0
$$

and

$$
\frac{\partial \tilde{v}_{0_{x}}}{\partial x}-\frac{\partial \tilde{v}_{1_{x}}}{\partial \eta} \frac{\partial \zeta_{0}}{\partial x}+\frac{\partial \tilde{v}_{0_{y}}}{\partial y}-\frac{\partial \tilde{v}_{1_{y}}}{\partial \eta} \frac{\partial \zeta_{0}}{\partial y}+\frac{\partial \tilde{v}_{1_{z}}}{\partial \eta}=0 .
$$

Differentiation of (3.26) with respect to $\eta$ gives

$$
\frac{\partial^{2} \tilde{v}_{1_{x}}}{\partial \eta^{2}} \frac{\partial \zeta_{0}}{\partial x}+\frac{\partial^{2} \tilde{v}_{1_{y}}}{\partial \eta^{2}} \frac{\partial \zeta_{0}}{\partial y}-\frac{\partial^{2} \tilde{v}_{1_{z}}}{\partial \eta^{2}}=0
$$

Multiplying the first equation of $(3.25)$ by $\frac{\partial \zeta_{0}}{\partial x}$, the second by $\frac{\partial \zeta_{0}}{\partial y}$, and the third by -1 and adding, we obtain

$$
P A\left(\frac{\partial^{2} \tilde{v}_{1_{x}}}{\partial \eta^{2}} \frac{\partial \zeta_{0}}{\partial x}+\frac{\partial^{2} \tilde{v}_{1_{y}}}{\partial \eta^{2}} \frac{\partial \zeta_{0}}{\partial y}-\frac{\partial^{2} \tilde{v}_{1_{z}}}{\partial \eta^{2}}\right)+A \frac{\partial \tilde{p}_{0}}{\partial \eta}=0
$$


It follows from this and (3.27) that

$$
\frac{\partial \tilde{p}_{0}}{\partial \eta}=0
$$

From (3.25) we then have

$$
\frac{\partial^{2} \tilde{v}_{1}}{\partial \eta^{2}}=0
$$

Using (3.18) and the boundedness of the solution, we obtain the continuity of the firstorder term of the outer expansion and of the first derivative of the zero-order term:

$$
\begin{gathered}
\left.\frac{\partial v_{0}}{\partial z_{1}}\right|_{z_{1}=+0}=\left.\frac{\partial v_{0}}{\partial z_{1}}\right|_{z_{1}=-0}, \\
\left.v_{1}\right|_{z_{1}=+0}=\left.v_{1}\right|_{z_{1}=-0} .
\end{gathered}
$$

Differentiating (3.15) twice and (3.12) once with respect to $\eta$ and using (3.24), (3.28), (3.29), we have:

$$
\begin{gathered}
t_{0} \frac{\partial^{2} \tilde{p}_{1}}{\partial \eta^{2}}+P A \frac{\partial^{3} \tilde{v}_{2}}{\partial \eta^{3}}=0 \\
\frac{\partial^{3} \tilde{v}_{2_{x}}}{\partial \eta^{3}} \frac{\partial \zeta_{0}}{\partial x}+\frac{\partial^{3} \tilde{v}_{2_{y}}}{\partial \eta^{3}} \frac{\partial \zeta_{0}}{\partial y}-\frac{\partial^{3} \tilde{v}_{2_{z}}}{\partial \eta^{3}}=0 .
\end{gathered}
$$

Multiplying the components of (3.31) respectively by $\frac{\partial \zeta_{0}}{\partial x}, \frac{\partial \zeta_{0}}{\partial y}$, and -1 and adding,

$$
P A\left(\frac{\partial^{3} \tilde{v}_{2_{x}}}{\partial \eta^{3}} \frac{\partial \zeta_{0}}{\partial x}+\frac{\partial^{3} \tilde{v}_{2_{y}}}{\partial \eta^{3}} \frac{\partial \zeta_{0}}{\partial y}-\frac{\partial^{3} \tilde{v}_{2_{z}}}{\partial \eta^{3}}\right)+A \frac{\partial^{2} \tilde{p}_{1}}{\partial \eta^{2}}=0
$$

Thus

$$
\frac{\partial^{2} \tilde{p}_{1}}{\partial \eta^{2}}=0
$$

and

$$
\frac{\partial^{3} \tilde{v}_{2}}{\partial \eta^{3}}=0
$$

From (3.19) and boundedness, the following jump conditions result:

$$
\begin{gathered}
\left.\frac{\partial^{2} v_{0}}{\partial z_{1}^{2}}\right|_{z_{1}=+0}=\left.\frac{\partial^{2} v_{0}}{\partial z_{1}^{2}}\right|_{z_{1}=-0}, \\
\left.\frac{\partial v_{1}}{\partial z_{1}}\right|_{z_{1}=+0}=\left.\frac{\partial v_{1}}{\partial z_{1}}\right|_{z_{1}=-0}, \\
\left.v_{2}\right|_{z_{1}=+0}=\left.v_{2}\right|_{z_{1}=-0} .
\end{gathered}
$$

Taking the third derivative of (3.16) and the second derivative of (3.14) with respect to $\eta$ and using (3.24), (3.28), (3.29), (3.33), and (3.34), we have

$$
\begin{array}{r}
t_{0} \frac{\partial^{3} \tilde{p}_{2}}{\partial \eta^{3}}+P A \frac{\partial^{4} \tilde{v}_{3}}{\partial \eta^{4}}+Q \gamma \frac{\partial^{2} \tilde{\theta}_{1}}{\partial \eta^{2}}=0 \\
\frac{\partial^{4} \tilde{v}_{3_{x}}}{\partial \eta^{4}} \frac{\partial \zeta_{0}}{\partial x}+\frac{\partial^{4} \tilde{v}_{3_{y}}}{\partial \eta^{4}} \frac{\partial \zeta_{0}}{\partial y}-\frac{\partial^{4} \tilde{v}_{3_{z}}}{\partial \eta^{4}}=0
\end{array}
$$


Proceeding as before, we get

$$
P A\left(\frac{\partial^{4} \tilde{v}_{3_{x}}}{\partial \eta^{4}} \frac{\partial \zeta_{0}}{\partial x}+\frac{\partial^{4} \tilde{v}_{3_{y}}}{\partial \eta^{4}} \frac{\partial \zeta_{0}}{\partial y}-\frac{\partial^{4} \tilde{v}_{3_{z}}}{\partial \eta^{4}}\right)+A \frac{\partial^{3} \tilde{p}_{2}}{\partial \eta^{3}}-Q \frac{\partial^{2} \tilde{\theta}_{1}}{\partial \eta^{2}}=0
$$

Thus from (3.37) and (3.36) we obtain

$$
A \frac{\partial^{3} \tilde{p}_{2}}{\partial \eta^{3}}-Q \frac{\partial^{2} \tilde{\theta}_{1}}{\partial \eta^{2}}=0
$$

and

$$
\gamma_{0} \frac{\partial^{2} \tilde{\theta}_{1}}{\partial \eta^{2}}=\frac{\partial^{4} \tilde{v}_{3}}{\partial \eta^{4}}
$$

with

$$
\gamma_{0}=\left(-\frac{\partial \zeta_{0}}{\partial x} \frac{R}{A^{2}},-\frac{\partial \zeta_{0}}{\partial y} \frac{R}{A^{2}}, \frac{R}{A^{2}}-\frac{R}{A}\right) .
$$

Integrating (3.38) with respect to $\eta$, and using (3.20), (3.21), and (3.22) we have

$$
\left.\frac{\partial^{3} v_{0}}{\partial z_{1}^{3}}\right|_{z_{1}=-0}-\left.\frac{\partial^{3} v_{0}}{\partial z_{1}^{3}}\right|_{z_{1}=+0}=-\left.\gamma_{0} \frac{\partial \theta_{0}}{\partial z_{1}}\right|_{z_{1}=+0}
$$

The equations (3.23), (3.30), (3.35), and (3.39) give the jump conditions for the velocity at the front.

Introduce

$$
s=\tilde{v}_{0_{x}} \frac{\partial \zeta_{0}}{\partial x}+\tilde{v}_{0_{y}} \frac{\partial \zeta_{0}}{\partial y}-\tilde{v}_{0_{z}} .
$$

From (3.9), (3.11), and (3.24) it follows that $\tilde{\alpha}_{0}$ is a monotonic function satisfying $0<$ $\tilde{\alpha}_{0}<1$. Since we only consider the zero-order reaction, $\phi\left(\tilde{\alpha}_{0}\right) \equiv 1$. Thus, multiplying (3.8) by $\frac{\partial \tilde{\theta}_{1}}{\partial \eta}$ and integrating, we obtain

$$
\left.\left(\frac{\partial \tilde{\theta}_{1}}{\partial \eta}\right)^{2}\right|_{\infty}-\left.\left(\frac{\partial \tilde{\theta}_{1}}{\partial \eta}\right)^{2}\right|_{-\infty}=2 A^{-1} \int_{-\infty}^{\theta_{1}} \exp (\tau /(1+\delta \tau)) d \tau
$$

Subtracting (3.8) from (3.9) and integrating, we have

$$
\left.\frac{\partial \tilde{\theta}_{1}}{\partial \eta}\right|_{\infty}-\left.\frac{\partial \tilde{\theta}_{1}}{\partial \eta}\right|_{-\infty}=-A^{-1}\left(\frac{\partial \zeta_{0}}{\partial t}+s\right) .
$$

Equations (3.40)-(3.41) give the jump conditions for the temperature across the front.

Using the matching conditions above and truncating the expansion [12] as

$$
\theta_{0} \approx \theta,\left.\left.\quad \theta_{1}\right|_{z_{1}=-0} \approx Z \theta\right|_{z_{1}=0}, \quad \zeta_{0} \approx \zeta, \quad v_{0} \approx v,
$$

we can rewrite the jump conditions as

$$
\begin{aligned}
& \left.\left(\frac{\partial \theta}{\partial z_{1}}\right)^{2}\right|_{+0}-\left.\left(\frac{\partial \theta}{\partial z_{1}}\right)^{2}\right|_{-0} \\
& \quad=2 Z\left(1+\left(\frac{\partial \zeta}{\partial x}\right)^{2}+\left(\frac{\partial \zeta}{\partial y}\right)^{2}\right)^{-1} \int_{-\infty}^{\left.\theta\right|_{0}} \exp \left(\tau /\left(Z^{-1}+\delta \tau\right)\right) d \tau
\end{aligned}
$$




$$
\begin{aligned}
\left.\frac{\partial \theta}{\partial z_{1}}\right|_{+0}-\left.\frac{\partial \theta}{\partial z_{1}}\right|_{-0}= & -\left(1+\left(\frac{\partial \zeta}{\partial x}\right)^{2}+\left(\frac{\partial \zeta}{\partial y}\right)^{2}\right)^{-1} \\
\times & \left(\frac{\partial \zeta}{\partial t}+\left.\left(v_{x} \frac{\partial \zeta}{\partial x}+v_{y} \frac{\partial \zeta}{\partial y}-v_{z}\right)\right|_{z_{1}=0}\right) \\
\left.v_{z}\right|_{z_{1}=+0} & =\left.v_{z}\right|_{z_{1}=-0}, \\
\left.\frac{\partial v_{z}}{\partial z_{1}}\right|_{z_{1}=+0} & =\left.\frac{\partial v_{z}}{\partial z_{1}}\right|_{z_{1}=-0} \\
\left.\frac{\partial^{2} v_{z}}{\partial z_{1}^{2}}\right|_{z_{1}=+0} & =\left.\frac{\partial^{2} v_{z}}{\partial z_{1}^{2}}\right|_{z_{1}=-0}
\end{aligned}
$$

and

$$
\begin{aligned}
\left.\frac{\partial^{3} v_{z}}{\partial z_{1}^{3}}\right|_{z_{1}=-0}-\left.\frac{\partial^{3} v_{z}}{\partial z_{1}^{3}}\right|_{z_{1}=+0}= & -R\left(1+\left(\frac{\partial \zeta}{\partial x}\right)^{2}+\left(\frac{\partial \zeta}{\partial y}\right)^{2}\right)^{-1} \\
& \times\left.\left(\left(1+\left(\frac{\partial \zeta}{\partial x}\right)^{2}+\left(\frac{\partial \zeta}{\partial y}\right)^{2}\right)^{-1}-1\right) \frac{\partial \theta}{\partial z_{1}}\right|_{z_{1}=+0}
\end{aligned}
$$

Note that we need only consider the matching condition for the component $v_{z}$ of the velocity since the other components are not used below.

4. The interface problem. We further study the following interface problem which approximates the original system $(2.5)-(2.8)$ :

$z>\zeta$ :

$$
\begin{gathered}
\frac{\partial \theta}{\partial t}+v \nabla \theta=\Delta \theta \\
\alpha=0 \\
\frac{\partial v}{\partial t}+(v \nabla) v=-\nabla p+P \Delta v+Q\left(\theta+\theta^{0}\right) \gamma \\
\operatorname{div} v=0
\end{gathered}
$$

$z<\zeta:$

$$
\begin{gathered}
\frac{\partial \theta}{\partial t}+v \nabla \theta=\Delta \theta \\
\alpha=1, \\
\frac{\partial v}{\partial t}+(v \nabla) v=-\nabla p+P \Delta v+Q\left(\theta+\theta^{0}\right) \gamma \\
\operatorname{div} v=0,
\end{gathered}
$$

$z=\zeta$

$$
\left.\theta\right|_{\zeta-0}=\left.\theta\right|_{\zeta+0}
$$




$$
\begin{aligned}
& \left.\frac{\partial \theta}{\partial z}\right|_{\zeta-0}-\left.\frac{\partial \theta}{\partial z}\right|_{\zeta+0}=\left(1+\left(\frac{\partial \zeta}{\partial x}\right)^{2}+\left(\frac{\partial \zeta}{\partial y}\right)^{2}\right)^{-1} \\
& \times\left(\frac{\partial \zeta}{\partial t}+\left.\left(v_{x} \frac{\partial \zeta}{\partial x}+v_{y} \frac{\partial \zeta}{\partial y}-v_{z}\right)\right|_{\zeta}\right) \\
& \left.\left(\frac{\partial \theta}{\partial z}\right)^{2}\right|_{\zeta-0}-\left.\left(\frac{\partial \theta}{\partial z}\right)^{2}\right|_{\zeta+0} \\
& =-2 Z\left(1+\left(\frac{\partial \zeta}{\partial x}\right)^{2}+\left(\frac{\partial \zeta}{\partial y}\right)^{2}\right)^{-1} \int_{-\infty}^{\left.\theta\right|_{\zeta}} \exp \left(\tau /\left(Z^{-1}+\delta \tau\right)\right) d \tau \\
& \left.v_{z}\right|_{\zeta-0}=\left.v_{z}\right|_{\zeta+0}, \\
& \left.\frac{\partial v_{z}}{\partial z}\right|_{\zeta-0}=\left.\frac{\partial v_{z}}{\partial z}\right|_{\zeta+0} \\
& \left.\frac{\partial^{2} v_{z}}{\partial z^{2}}\right|_{\zeta-0}=\left.\frac{\partial^{2} v_{z}}{\partial z^{2}}\right|_{\zeta+0} \\
& \left.\frac{\partial^{3} v_{z}}{\partial z^{3}}\right|_{\zeta-0}-\left.\frac{\partial^{3} v_{z}}{\partial z^{3}}\right|_{\zeta+0}=-R\left(1+\left(\frac{\partial \zeta}{\partial x}\right)^{2}+\left(\frac{\partial \zeta}{\partial y}\right)^{2}\right)^{-1} \\
& \times\left.\left(\left(1+\left(\frac{\partial \zeta}{\partial x}\right)^{2}+\left(\frac{\partial \zeta}{\partial y}\right)^{2}\right)^{-1}-1\right) \frac{\partial \theta}{\partial z}\right|_{\zeta+0}
\end{aligned}
$$

with the conditions at infinity:

$$
z=-\infty: \theta=0, v=0, \quad z=+\infty: \theta=-1, v=0 .
$$

5. Linear stability analysis. In this section we present the linear stability analysis of the steady-state solution for the interface problem (4.1)-(4.16).

This problem has a traveling wave solution:

$$
(\theta(x, y, z, t), \alpha(x, y, z, t), v)=\left(\theta_{s}(z-u t), \alpha_{s}(z-u t), 0\right)
$$

where

$$
\begin{gathered}
\left(\theta_{s}\left(z_{2}, t\right), \alpha_{s}\left(z_{2}, t\right)\right)= \begin{cases}(0,1), & z_{2}<0 \\
\left(\exp \left(-u z_{2}\right)-1,1\right), & z_{2}>0\end{cases} \\
z_{2}=z-u t \quad \text { for } u=c .
\end{gathered}
$$

This solution will be referred to as a basic solution. It is a stationary solution of (4.1)(4.16) written in the moving coordinates, where equations (4.1), (4.5) and (4.3), (4.7) are replaced by

$$
\frac{\partial \theta}{\partial t}+v \nabla \theta=\Delta \theta+u \frac{\partial \theta}{\partial z_{2}}
$$


and

$$
\frac{\partial v}{\partial t}+(v \nabla) v=-\nabla p+P \Delta v+u \frac{\partial v}{\partial z_{2}}+Q\left(\theta+\theta^{0}\right) \gamma
$$

respectively, and all other equations remain unchanged.

We look for a solution of the form of the perturbed stationary solution:

$$
\theta=\theta_{s}+\tilde{\theta}, \quad p=p_{s}+\tilde{p}, \quad v=v_{s}+\tilde{v}
$$

Substituting (5.4) into (5.2)-(5.3), for the first-order terms we obtain:

$z_{2}>\xi$ :

$$
\begin{gathered}
\frac{\partial \tilde{\theta}}{\partial t}=\Delta \tilde{\theta}+u \frac{\partial \tilde{\theta}}{\partial z_{2}}-\tilde{v}_{z} \theta_{s}^{\prime}, \\
\frac{\partial \tilde{v}}{\partial t}=-\nabla \tilde{p}+P \Delta \tilde{v}+u \frac{\partial \tilde{v}}{\partial z_{2}}+Q \tilde{\theta}_{\gamma}, \\
\operatorname{div} \tilde{v}=0
\end{gathered}
$$

$z_{2}<\xi$

$$
\begin{gathered}
\frac{\partial \tilde{\theta}}{\partial t}=\Delta \tilde{\theta}+u \frac{\partial \tilde{\theta}}{\partial z_{2}} \\
\frac{\partial \tilde{v}}{\partial t}=-\nabla \tilde{p}+P \Delta \tilde{v}+u \frac{\partial \tilde{v}}{\partial z_{2}}+Q \tilde{\theta}_{\gamma} \\
\operatorname{div} \tilde{v}=0
\end{gathered}
$$

with $\xi=\zeta-u t$.

We denote

$$
(\tilde{\theta}, \tilde{v})= \begin{cases}\left(\hat{\theta}_{1}, \hat{v}_{1}\right) & \text { for } z_{2}<\xi \\ \left(\hat{\theta}_{2}, \hat{v}_{2}\right) & \text { for } z_{2}>\xi\end{cases}
$$

We linearize now the jump conditions (4.9)-(4.15). Taking into account that

$$
\begin{gathered}
\left.\theta\right|_{\xi \pm 0}=\theta_{s}( \pm 0)+\xi \theta_{s}^{\prime}( \pm 0)+\tilde{\theta}( \pm 0) \\
\left.\frac{\partial \theta}{\partial z_{2}}\right|_{\xi \pm 0}=\theta_{s}^{\prime}( \pm 0)+\xi \theta_{s}^{\prime \prime}( \pm 0)+\left.\frac{\partial \tilde{\theta}}{\partial z_{2}}\right|_{\xi \pm 0}
\end{gathered}
$$


up to the high-order terms, we obtain

$$
\begin{aligned}
& \left.\hat{\theta}_{2}\right|_{z_{2}=0}-\left.\hat{\theta}_{1}\right|_{z_{2}=0}=u \xi, \\
& \left.\frac{\partial \hat{\theta}_{2}}{\partial z_{2}}\right|_{0}-\left.\frac{\partial \hat{\theta}_{1}}{\partial z_{2}}\right|_{z_{2}=0}=-u \xi^{2}-\frac{\partial \xi}{\partial t}+\left.\tilde{v}_{z}\right|_{z_{2}=0} \\
& u^{2} \xi+\left.\frac{\partial \hat{\theta}_{2}}{\partial z_{2}}\right|_{z_{2}=0}=-\left.\frac{Z}{u} \frac{\partial \hat{\theta}_{1}}{\partial z_{2}}\right|_{z_{2}=0}, \\
& \left.\hat{v}_{2_{z}}\right|_{z_{2}=0}=\left.\hat{v}_{1_{z}}\right|_{z_{2}=0}, \\
& \left.\frac{\partial \hat{v}_{2_{z}}}{\partial z_{2}}\right|_{z_{2}=0}=\left.\frac{\partial \hat{v}_{1_{z}}}{\partial z_{2}}\right|_{z_{2}=0}, \\
& \left.\frac{\partial^{2} \hat{v}_{2_{z}}}{\partial z_{2}^{2}}\right|_{z_{2}=0}=\left.\frac{\partial^{2} \hat{v}_{1_{z}}}{\partial z_{2}^{2}}\right|_{z_{2}=0}, \\
& \left.\frac{\partial^{3} \hat{v}_{2_{z}}}{\partial z_{2}^{3}}\right|_{z_{2}=0}=\left.\frac{\partial^{3} \hat{v}_{1_{z}}}{\partial z_{2}^{3}}\right|_{z_{2}=0} .
\end{aligned}
$$

Consider perturbations in the form

$$
\begin{array}{cc}
\hat{\theta}_{i}=\theta_{i}\left(z_{2}\right) \exp \left(\omega t+i\left(k_{1} x+k_{2} y\right)\right), & i=1,2, \\
\hat{v}_{i_{z}}=v_{i}\left(z_{2}\right) \exp \left(\omega t+i\left(k_{1} x+k_{2} y\right)\right), & i=1,2, \\
\xi=\varepsilon_{1} \exp \left(\omega t+i\left(k_{1} x+k_{2} y\right)\right) . &
\end{array}
$$

Here $\varepsilon_{1}$ is the amplitude, $\omega$ the frequency, and $k_{1}, k_{2}$ the wavenumbers. Substituting (5.16) $-(5.18)$ into (5.9)-(5.15), we have

$$
\begin{gathered}
\theta_{2}(0)-\theta_{1}(0)=\varepsilon_{1} u, \\
\theta_{2}^{\prime}(0)-\theta_{1}^{\prime}(0)=-\varepsilon_{1}\left(\omega+u^{2}\right)+v_{1}(0), \\
u^{2} \varepsilon_{1}+\theta_{2}^{\prime}(0)=-\frac{Z}{u} \theta_{1}(0), \\
v_{2}^{(i)}(0)=v_{1}^{(i)}(0), \quad i=0,1,2,3,
\end{gathered}
$$

where

$$
v^{(i)}=\frac{\partial^{i} v}{\partial z^{i}}, \quad \theta^{\prime}=\frac{\partial \theta}{\partial z} .
$$

To find the perturbation terms we apply the transformation rotrot to equations (5.6), (5.8). This allows us to eliminate the pressure and to consider only the velocity component $v_{z}$. Equations (5.6), (5.8) become

$$
\frac{\partial}{\partial t} \Delta \tilde{v}_{z}-\frac{\partial}{\partial z_{2}} \Delta \tilde{v}_{z}=P \Delta \Delta \tilde{v}_{z}+Q \Delta_{1} \tilde{\theta}
$$

for $z_{2}>\xi$ and $z_{2}<\xi$. Substituting (5.1), (5.17), and (5.18) into (5.5), (5.7), and (5.24), we have

$$
\omega\left(v_{i}^{\prime \prime}-k^{2} v_{i}\right)-u\left(v_{2}^{\prime \prime \prime}-k^{2} v_{i}^{\prime}\right)=P\left(v_{2}^{\prime \prime \prime \prime}-k^{2} v_{i}^{\prime \prime}+k^{4} v_{i}\right)-Q k^{2} \theta_{i}, \quad i=1,2,
$$




$$
\begin{gathered}
\omega \theta_{2}-u \theta_{2}^{\prime}+v \theta_{s}^{\prime}=\theta_{2}^{\prime \prime}-k^{2} \theta_{2}, \\
\omega \theta_{1}-u \theta_{1}^{\prime}=\theta_{1}^{\prime \prime}-k^{2} \theta_{1},
\end{gathered}
$$

where $k^{2}=k_{1}^{2}+k_{2}^{2}$. Introducing the linear differential operators defined by

$$
\begin{gathered}
L_{1} v=P v^{\prime \prime \prime \prime}+u v^{\prime \prime \prime}-\left(2 P k^{2}+\omega\right) v^{\prime \prime}-u k^{2} v^{\prime}+k^{2}\left(P k^{2}+\omega\right) v, \\
L_{2} \theta=\theta^{\prime \prime}+u \theta^{\prime}-\left(k^{2}+\omega\right) \theta,
\end{gathered}
$$

we write Eqs. (5.25)-(5.27) as

$$
\begin{gathered}
L_{1} v_{i}=Q k^{2} \theta_{i}, \quad i=1,2, \\
L_{2} \theta_{2}=-u e^{-u z_{2}} v_{2}, \quad L_{2} \theta_{1}=0 .
\end{gathered}
$$

Recall that the perturbations decay at infinity; so $v( \pm \infty)=0, \theta( \pm \infty)=0$. The general solutions of (5.28) and (5.29) are

$$
\begin{gathered}
\theta_{1}\left(z_{2}\right)=\tilde{b}_{1} \tilde{s}_{1}\left(z_{2}\right), \quad \theta_{2}\left(z_{2}\right)=b_{1} s_{1}\left(z_{2}\right)+b_{2} s_{2}\left(z_{2}\right)+b_{3} s_{3}\left(z_{2}\right), \\
v_{1}\left(z_{2}\right)=\tilde{b}_{1} \tilde{w}_{1}\left(z_{2}\right)+\tilde{b}_{2} \tilde{w}_{2}\left(z_{2}\right)+\tilde{b}_{3} \tilde{w}_{3}\left(z_{2}\right), \quad v_{2}\left(z_{2}\right)=b_{1} w_{1}\left(z_{2}\right)+b_{2} w_{2}\left(z_{2}\right)+b_{3} w_{3}\left(z_{2}\right),
\end{gathered}
$$

where $b_{1}, b_{2}, b_{3}, \tilde{b}_{1}, \tilde{b}_{2}, \tilde{b}_{3}$ are arbitrary constants, and

$$
\begin{gathered}
\tilde{w}_{i}\left(z_{2}\right)=\tilde{a}_{i} e^{\tilde{\sigma}_{i} z_{2}}, \quad i=1,2,3, \quad \tilde{s}_{1}\left(z_{2}\right)=e^{\tilde{\sigma}_{1} z_{2}} \\
w_{i}=\sum_{j=1}^{\infty} a_{i j} e^{\sigma_{i j} z_{2}}, \quad s_{i}=\sum_{j=1}^{\infty} c_{i j} e^{\sigma_{i j} z_{2}}, \quad i=1,2,3, \\
\tilde{\sigma}_{1}=-\frac{u}{2}+\sqrt{\frac{u^{2}}{4}+k^{2}+\omega}, \quad \tilde{\sigma}_{2}=+k, \quad \tilde{\sigma}_{3}=-\frac{u}{2 P}+\sqrt{\frac{u^{2}}{4 P^{2}}+k^{2}+\frac{\omega}{P}} \\
\sigma_{11}=-\frac{u}{2}-\sqrt{\frac{u^{2}}{4}+k^{2}+\omega}, \quad \sigma_{21}=-k, \quad \sigma_{31}=-\frac{u}{2 P}-\sqrt{\frac{u^{2}}{4 P^{2}}+k^{2}+\frac{\omega}{P}}
\end{gathered}
$$

where $k$ is definitely supposed to be positive, and

$$
\begin{aligned}
& \sigma_{i j}=\sigma_{i, j-1}-u, \quad i=1,2,3, j=2,3, \ldots, \\
& c_{11}=1, \quad c_{21}=c_{31}=0, \quad a_{21}=a_{31}=1 \text {, } \\
& \tilde{a}_{2}=1, \quad \tilde{a}_{3}=1, \\
& a_{1 j}=\frac{Q k^{2} c_{1 j}}{\left(\sigma_{1 j}^{2}-k^{2}\right)\left(P \sigma_{1 j}^{2}+u \sigma_{1 j}-\left(P k^{2}+\omega\right)\right)}, \quad j=1,2,3, \ldots, \\
& \tilde{a}_{1}=\frac{Q k^{2}}{\left(\tilde{\sigma}_{1}^{2}-k^{2}\right)\left(P \tilde{\sigma}_{1}^{2}+u \tilde{\sigma}_{1}-\left(P k^{2}+\omega\right)\right)}, \\
& c_{i, j+1}=\frac{-u a_{i j}}{\sigma_{i, j+1}^{2}+u \sigma_{i, j+1}-\left(k^{2}+\omega\right)}, \quad i=1,2,3, j=1,2,3, \ldots \text {, } \\
& a_{i, j+1}=\frac{Q k^{2} c_{i, j+1}}{\left(\sigma_{i, j+1}^{2}-k^{2}\right)\left(P \sigma_{i, j+1}^{2}+u \sigma_{i, j+1}-\left(P k^{2}+\omega\right)\right)}, \quad i=2,3, j=1,2,3, \ldots
\end{aligned}
$$


We assume that

$$
\begin{array}{r}
\left(\tilde{\sigma}_{1}^{2}-k^{2}\right)\left(P \tilde{\sigma}_{1}^{2}+u \tilde{\sigma}_{1}-\left(P k^{2}+\omega\right)\right) \neq 0, \\
\left(\sigma_{i j}^{2}-k^{2}\right)\left(P \sigma_{i j}^{2}+u \sigma_{i j}-\left(P k^{2}+\omega\right)\right) \neq 0,
\end{array}
$$

for $i=1$ and $j=1,2,3, \ldots$ and for $i=2,3$, and $j=2,3, \ldots$ that

$$
\sigma_{i j}^{2}+u \sigma_{i j}-\left(k^{2}+\omega\right) \neq 0
$$

for $i=1,2,3$ and $j=2,3, \ldots$.

Note that the series (5.33) converge, and the solutions $\left(w_{i}, s_{i}\right), i=1,2,3$ are linearly independent if the numbers $\sigma_{i 1}$ are different, i.e., $P \neq 1$ and $P \neq \infty$ (see [6]).

6. The dispersion relation. In this section we determine the stability boundary. For this we substitute $\theta_{i}, v_{i}, i=1,2$, as given by (5.30), (5.31), into the matching conditions (5.20)-(5.23). We obtain a system of seven linear homogeneous algebraic equations with the seven unknowns $b_{1}, b_{2}, b_{3}, \tilde{b}_{1}, \tilde{b}_{2}, \tilde{b}_{3}, \varepsilon_{1}$. To obtain a nontrivial solution of this system requires the dispersion relation, i.e., an equality that includes the eigenvalue $\omega$ and various parameters of the problem. If $\omega$ lies on the imaginary axis, the dispersion relation determines the stability boundary. It is the cellular stability boundary if $\omega=0$, and the oscillatory stability boundary if $\omega=i \phi$ for $\phi \neq 0$.

Some analysis of the dispersion relation can be obtained without a concrete form for the perturbations. Denote

$$
d=\sqrt{1+\frac{4 \omega}{u^{2}}+\frac{4 k^{2}}{u^{2}}}
$$

so that

$$
\tilde{\sigma}_{1}=\frac{u}{2}(d-1) \quad \text { and } \quad \theta_{1}\left(z_{2}\right)=\tilde{b}_{1} e^{\tilde{\sigma}_{1} z_{2}} .
$$

We now assume that $\omega \neq 0$ and $\tilde{b}_{1} \neq 0$. Eliminating $\varepsilon_{1}$ from (5.20)-(5.21) we have

$$
\frac{1}{u} \frac{\theta_{1}^{\prime}(0)}{\theta_{1}(0)}-\frac{1}{u} \frac{\theta_{2}^{\prime}(0)}{\theta_{1}(0)}+\frac{1}{u} \frac{v_{1}(0)}{\theta_{1}(0)}=\frac{\omega+u^{2}}{u^{2}}\left(\frac{\theta_{2}(0)}{\theta_{1}(0)}-1\right)
$$

and

$$
-\frac{1}{u} \frac{\theta_{1}^{\prime}(0)}{\theta_{1}(0)}+\frac{\omega}{u^{2}}\left(\frac{\theta_{2}(0)}{\theta_{1}(0)}-1\right)-\frac{1}{u} \frac{v_{1}(0)}{\theta_{1}(0)}=\frac{Z}{u^{2}} .
$$

Let us introduce the following notation:

$$
\begin{array}{clrl}
\sigma_{+}=\frac{1}{u} \frac{\theta_{2}^{\prime}(0)}{\theta_{2}(0)}, & \sigma_{-}=\frac{1}{u} \frac{\theta_{1}^{\prime}(0)}{\theta_{1}(0)}, & \sigma_{z}=\frac{1}{u} \frac{v_{1}(0)}{\theta_{1}(0)}, \\
\sigma_{1}=\frac{\theta_{2}(0)}{\theta_{1}(0)}, & \omega_{1}=\frac{\omega}{u^{2}}, & Z_{1}=\frac{Z}{u^{2}}
\end{array}
$$

assuming that $\theta_{2}(0) \neq 0$. Then, from $(6.1)-(6.2)$,

$$
\begin{gathered}
\sigma_{-}-\sigma_{+} \sigma_{1}+\sigma_{z}=\left(\omega_{1}+1\right)\left(\sigma_{1}-1\right), \\
-\sigma_{-}+\omega_{1}\left(\sigma_{1}-1\right)-\sigma_{z}=Z_{1} .
\end{gathered}
$$


We now express $\sigma_{1}$ from (6.4) and substitute into (6.3) to obtain

$$
-\sigma_{+}\left(Z_{1}+\omega_{1}+\frac{d-1}{2}+\sigma_{z}\right)=\omega_{1} Z_{1}+Z_{1}+\frac{d-1}{2}+\sigma_{z} .
$$

This equation includes the eigenvalue $\omega_{1}$ and the functions $\sigma_{+}$and $\sigma_{z}$ which can be found from the equations for the perturbations, i.e., $\sigma_{+}=\sigma_{+}(R, \omega, k, P, u), \sigma_{z}=$ $\sigma_{z}(R, \omega, k, P, u)$ (see section 5).

We now consider some limiting cases for the general dispersion relation (6.5):

(i) If the product of the reaction is a solid, the perturbation of the velocity is zero for $z_{2}<\xi$; so the continuity of the velocity implies that $v_{1}(0)=0$ (i.e., that $\sigma_{z}=0$ ). In this case the dispersion relation (6.5) coincides with that for the problem with a solid product $[6]$ :

$$
-\sigma_{+}\left(Z_{1}+\omega_{1}+\frac{d-1}{2}\right)=Z_{1}+\frac{d-1}{2}+\omega_{1} Z_{1} .
$$

(ii) If the Rayleigh number is zero, then the perturbation of the velocity is zero for $z_{2}<\xi$ and $z_{2}>\xi$. In this case we have

$$
\theta_{2}=c_{2} e^{\mu_{2} z_{2}}, \quad \mu_{2}=-\frac{u}{2}(d+1) .
$$

Thus

$$
\sigma_{+}=-\frac{1+d}{2}, \quad \sigma_{z}=0
$$

and (6.5) becomes

$$
2 Z_{1}\left(2 \omega_{1}+1-d\right)=2 \omega_{1}(1+d)+(1-d)^{2} .
$$

This dispersion relation coincides with that for the condensed phase combustion [1].

6.1. Oscillatory stability boundary. To find the oscillatory stability boundary, we substitute $\omega=i \phi, \phi \neq 0$ and $\theta_{i}, v_{i}, i=1,2$ given by (5.30), (5.31) in (5.20)-(5.23). As was mentioned above, we obtain a linear algebraic system of seven equations with seven unknowns $b_{i}, \tilde{b}_{i}, i=1,3$ and $\varepsilon_{1}$. The condition for nontrivial solvability of this system is a zero determinant. We note that it is a complex equation. Separating real and imaginary parts, we obtain two real equations. From them we find the unknown frequency $\phi$ and the values of parameters that give the stability boundary. We finally note that the dispersion relation is very complicated and that it can only be analyzed numerically.

Figure 1 shows the critical values of the Zeldovich number as a function of the wavenumber for different values of the Rayleigh number that give the oscillatory stability boundary. The front propagating upward $(R>0)$ appears to be more unstable with respect to multidimensional perturbations $(k \neq 0)$ than in the pure combustion problem without hydrodynamics $(R=0)$; the front propagating downward $(R<0)$ is more stable.

We recall that to find the stability boundary we used only the component $\tilde{v}_{z}$ of the perturbed velocity. When this component is known, the two others can easily be found. Indeed, from the third equation in (5.6) and (5.8) we find the perturbation of the pressure. Substituting it into the second equation in (5.6) and (5.8), we find $\tilde{v}_{y}$. Then $\tilde{v}_{x}$ can be found from the incompressibility condition, $\operatorname{div} \tilde{v}=0$. 


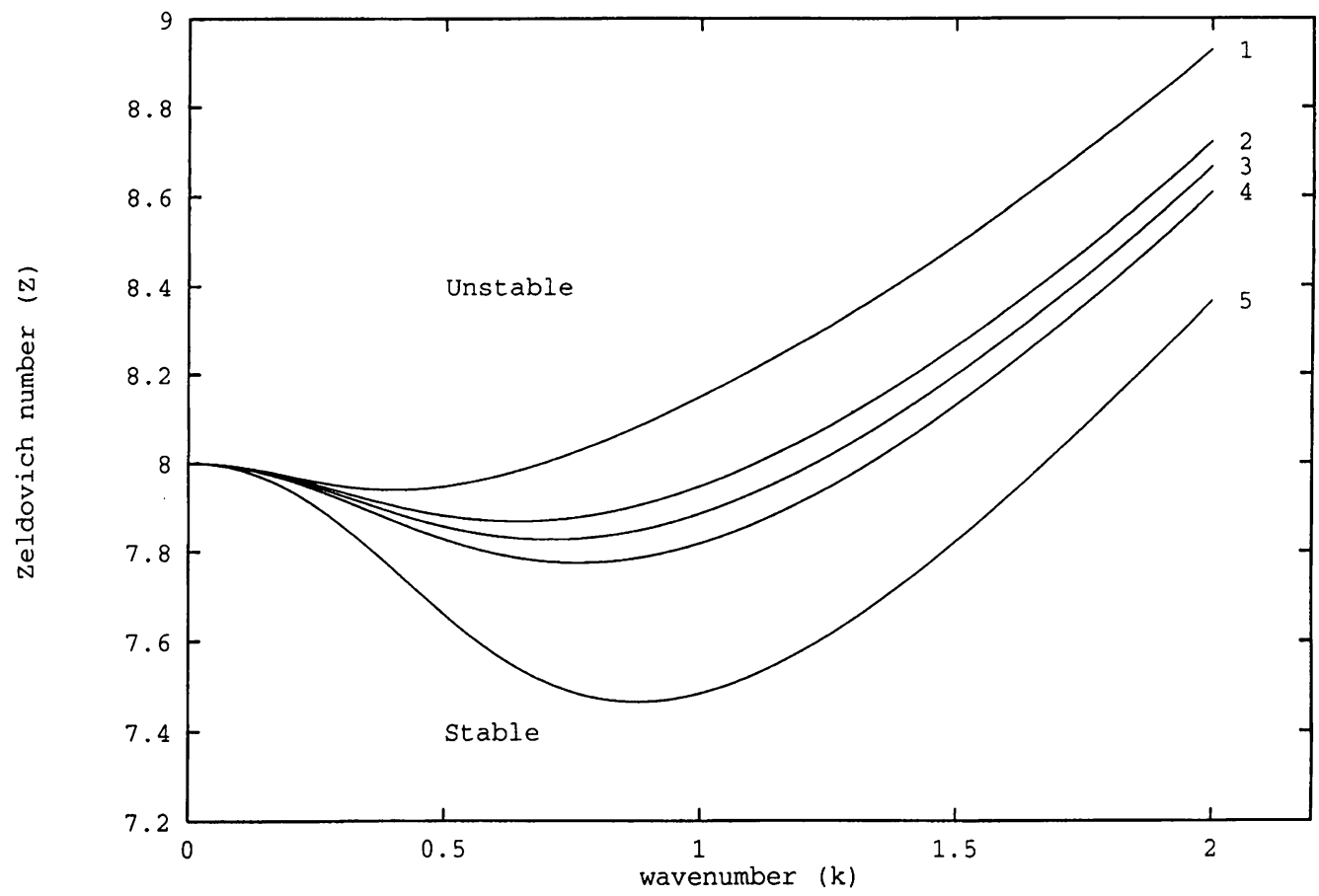

FIG. 1. Critical value of $Z$ as a function of the wavenumber $k$ for different values of $R ; P=0.5, u=\sqrt{2}$, (1) $R=-50$, (2) $R=-10$, (3) $R=0$, (4) $R=10$, (5) $R=50$

Consider, for simplicity, the two-dimensional case. We have for $j=1,2$ :

$$
\hat{\theta}_{j}=\theta_{j}\left(z_{2}\right) \exp \left(\omega t+i k_{1} x\right), \quad \hat{v}_{j z}=v_{j}\left(z_{2}\right) \exp \left(\omega t+i k_{1} x\right), \quad \omega=i \phi .
$$

Here $j=1$ corresponds to $\xi>z_{2}$ and $j=2$ to $\xi<z_{2}$. Denote by $\psi_{j}$ the stream function. Then

$$
\hat{v}_{j z}=-\frac{\partial \psi_{j}}{\partial x}, \quad \hat{v}_{j x}=\frac{\partial \psi_{j}}{\partial z_{2}}
$$

We find $\psi_{j}$ from the first equality in (6.1.1),

$$
\psi_{j}\left(x, z_{2}\right)=\frac{i}{k_{1}} v_{j}\left(z_{2}\right) \exp \left(\omega t+i k_{1} x\right)
$$

and $\tilde{v}_{j x}$ from the second equality,

$$
\tilde{v}_{j x}\left(x, z_{2}\right)=\frac{i}{k_{1}} \frac{\partial v_{j}\left(z_{2}\right)}{\partial z_{2}} \exp \left(\omega t+i k_{1} x\right) .
$$

We note that along with the functions $\tilde{\theta}$ and $\tilde{v}$, their complex conjugates and, consequently, the functions $\operatorname{Re}(\tilde{\theta})$ and $\operatorname{Re}(\tilde{v})$ also satisfy Eq. (5.24).

Figures $2 \mathrm{a}, 3 \mathrm{a}, 4 \mathrm{a}, 5 \mathrm{a}, 5 \mathrm{~b}$, and $5 \mathrm{c}$ (see pp. 18-22) show the velocity field found as the level lines of the real part of the stream function for the ascending and descending fronts, for oscillatory (pulsating) stability boundary in the space of parameters. Also, the perturbation of the temperature and the component $\tilde{v}_{z}$ of the velocity as functions 


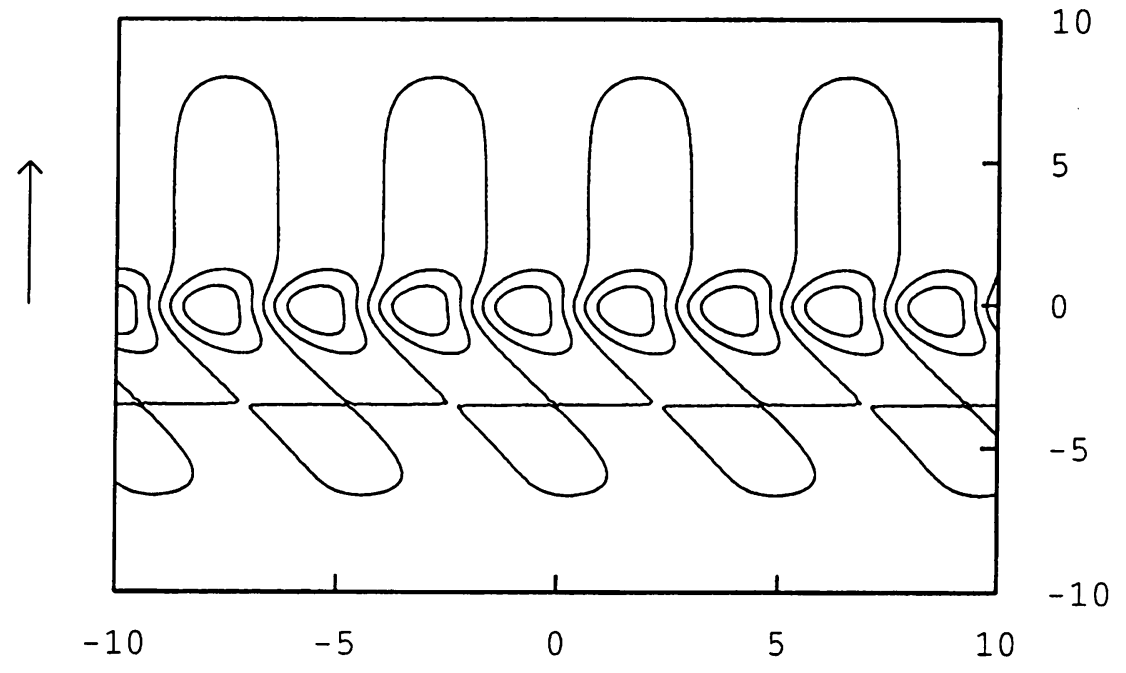

FIG. 2a. The streamlines of the perturbed velocity field (ascending front) (here and in the other figures the arrow shows the direction of the front propagation) for $R=10, k=1.34, Z=8, P=0.5$, $u=\sqrt{2}, \phi=7.0788$

of $z$ are shown in Figs. 2b, 3b, and $4 \mathrm{~b}$ (see pp. 19-21). Figures 5a and 5b (see pp. 21 and 22) show the influence of the Prandtl number on the form of the streamlines of the perturbed velocity field.

In the case of a solid product the similar functions are sketched in Figs. 6a, 6b, 7a, and $7 \mathrm{~b}$ (see pp. 23 and 24 ).

6.2. Cellular stability boundary. To find the cellular stability boundary, we put $\omega=0$. Then Eqs. (5.20)-(5.23) become

$$
\begin{gathered}
\theta_{2}^{\prime}(0)+u \theta_{2}(0)-\theta_{1}^{\prime}(0)-u \theta_{1}(0)-v_{1}(0)=0, \\
v_{2}^{(i)}(0)=v_{1}^{(i)}(0), \quad i=0,1,2,3, \\
Z_{1}=-\frac{d-1}{2}-\sigma_{z} .
\end{gathered}
$$

As before the solvability condition gives the stability boundary.

The critical value of the Zeldovich number as a function of the wavenumber is shown in Fig. 8 (see p. 25).

We note that not only ascending fronts $(R>0)$ but also descending fronts $(R<0)$ can be unstable (Fig. 9 on p. 26). This is a principal difference between the problem under consideration and the case of a solid product [6] where the cellular instability for a descending front cannot occur. Another difference is that the stability boundary in the case of a liquid product depends on the value of the Zeldovich number. Increasing $Z$ makes the front propagating upward more stable and the front propagating downward less stable.

Figure 10 (see p. 27) shows the critical value of the Rayleigh number found numerically as a function of the wavenumber for different values of the Prandtl number. We conclude 


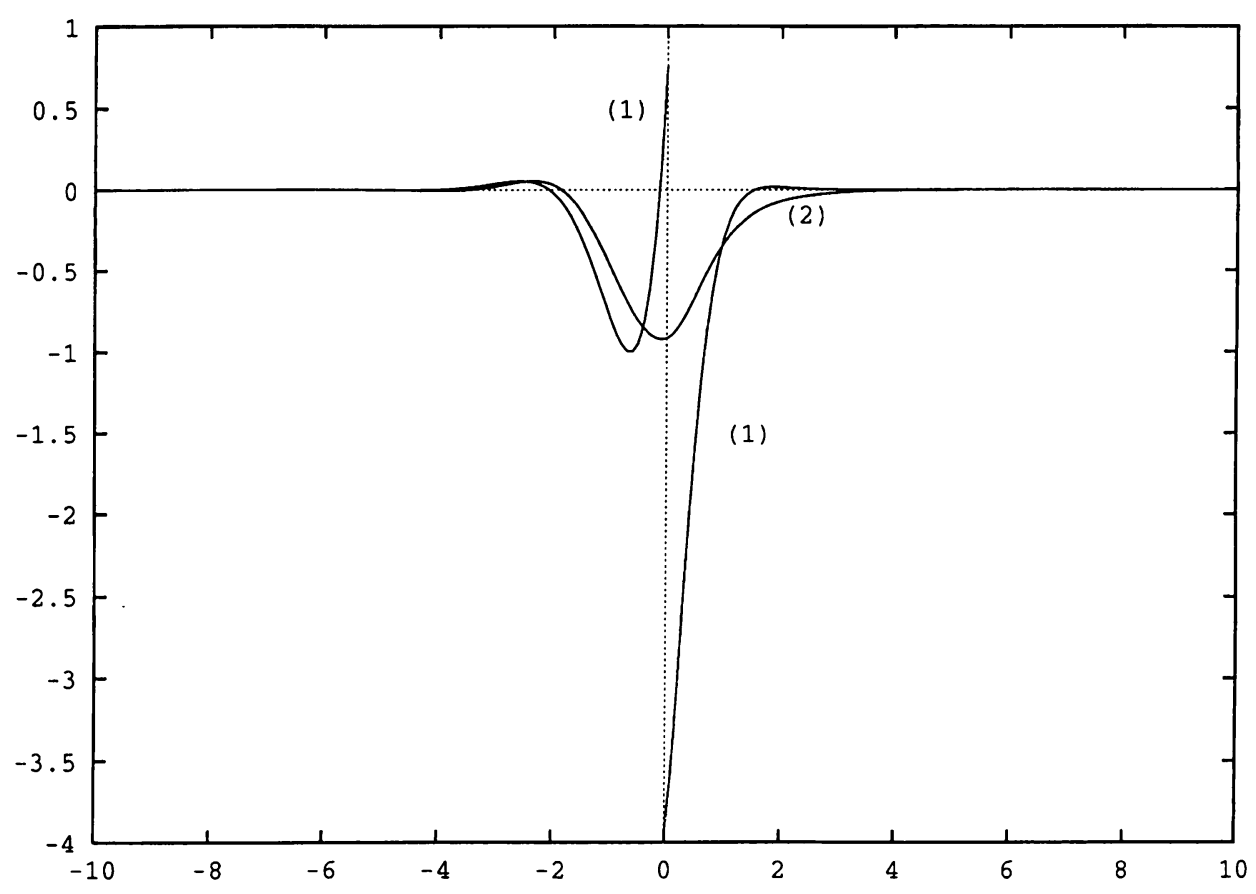

FIG. 2b. (1) $\tilde{\theta}$ as a function of the variable $z$ (ascending front), (2) $\tilde{v}_{z}$ as a function of $z$ for $R=10, k=1.34, Z=8, P=0.5, u=\sqrt{2}$, $\phi=7.0788$

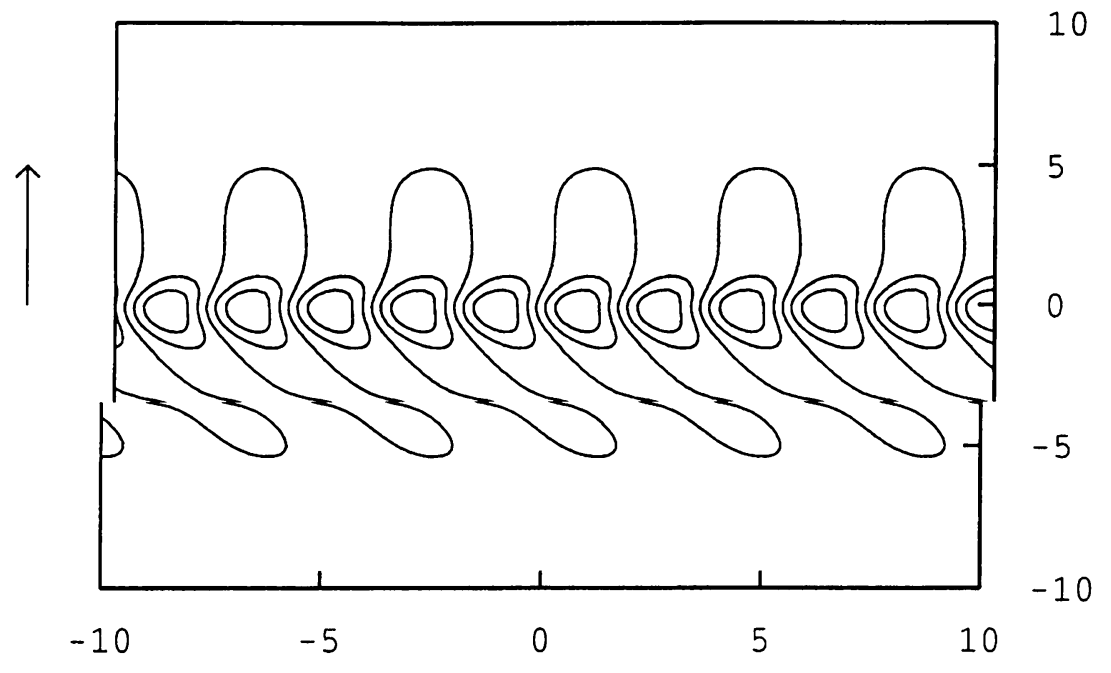

FIG. 3a. The streamlines of the perturbed velocity field (ascending front) for $R=50, k=1.68, Z=8, P=0.5, u=\sqrt{2}, \phi=8.2011$

that for ascending as well as for descending fronts, decrease of $P$ makes the front more stable. 


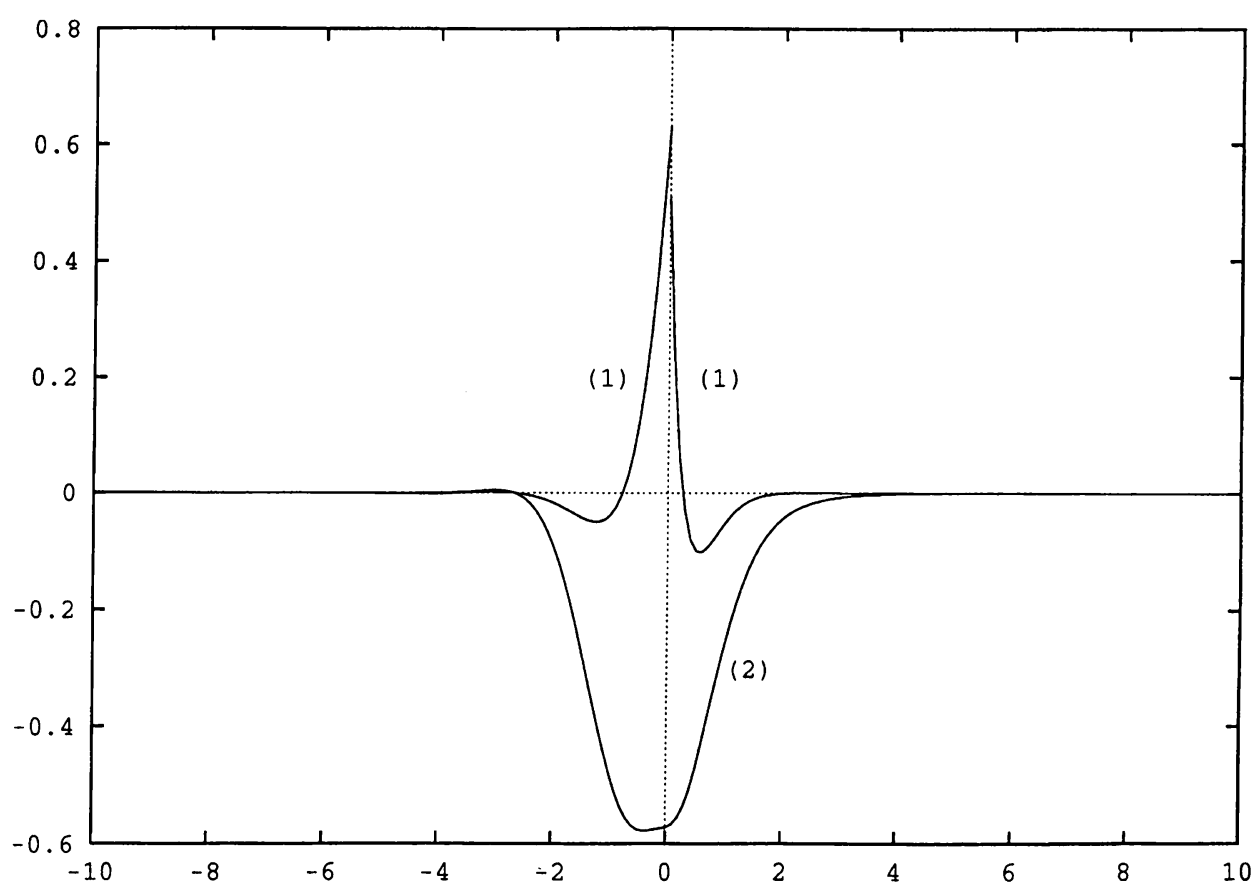

FIG. 3b. (1) $\tilde{\theta}$ as a function of the variable $z$ (ascending front), (2) $\tilde{v}_{z}$ as a function of $z$ for $R=50, k=1.68, Z=8, P=0.5, u=\sqrt{2}$, $\phi=8.2011$

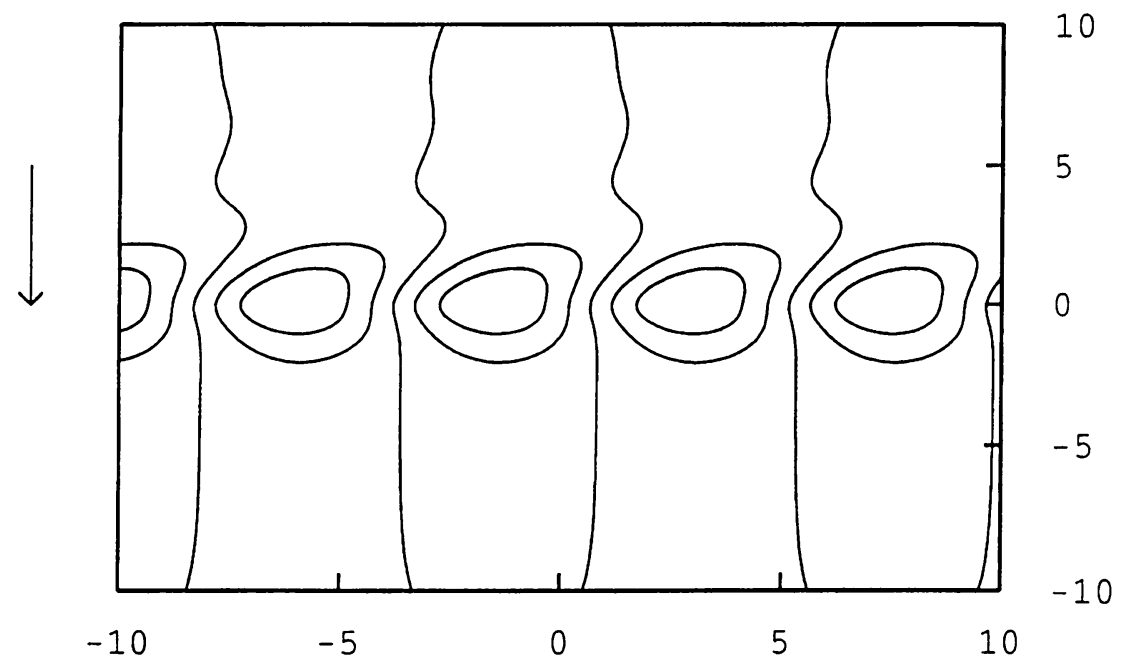

FIG. 4a. The streamlines of the perturbed velocity field (descending front) for $R=-50, k=0.7, Z=8, P=0.5, u=\sqrt{2}, \phi=5.03054$ 


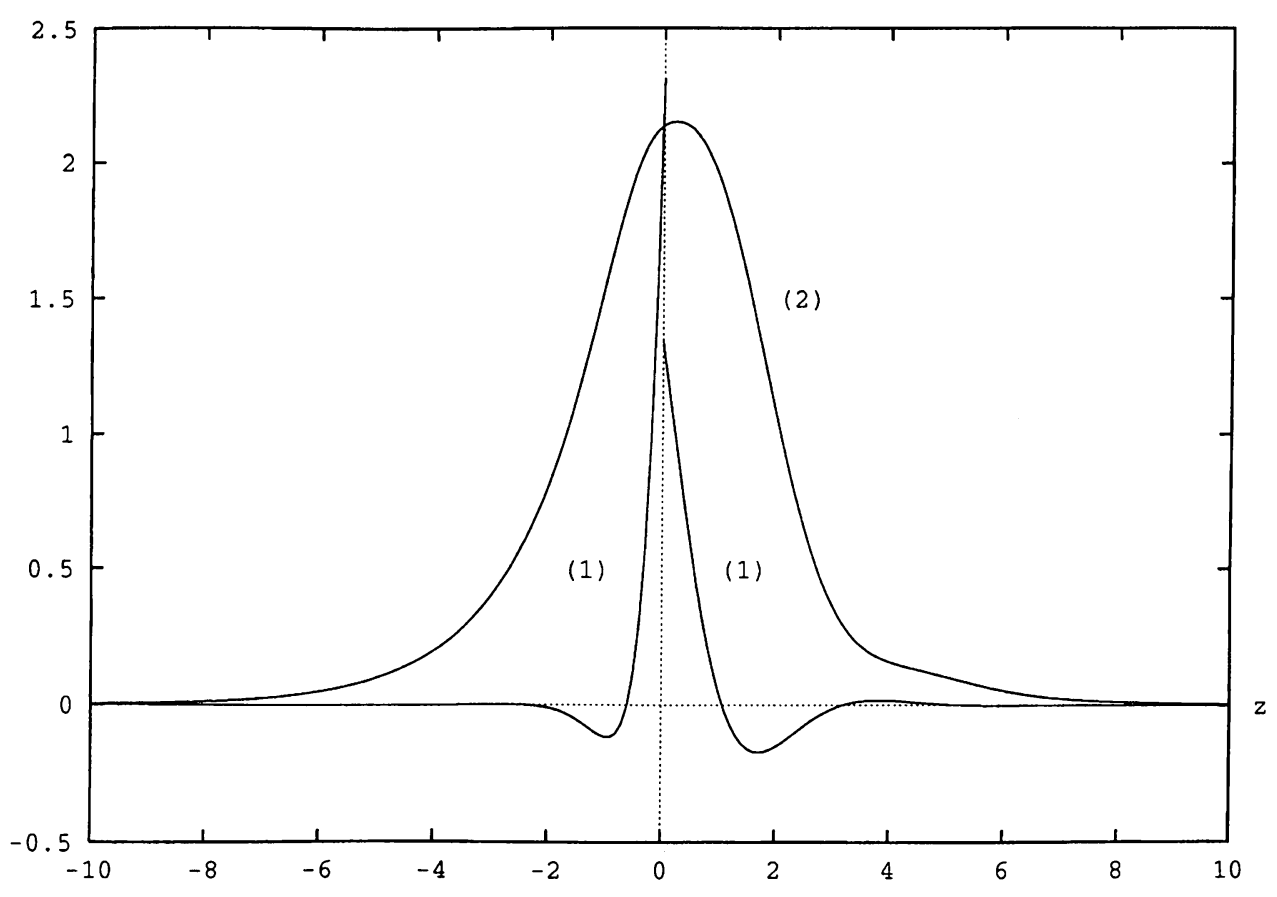

FIG. 4b. (1) $\tilde{\theta}$ as a function of the variable $z$ (descending front), (2) $\tilde{v}_{z}$ as a function of $z$ for $R=-50, k=0.7, Z=8, P=0.5, u=\sqrt{2}$, $\phi=5.03054$

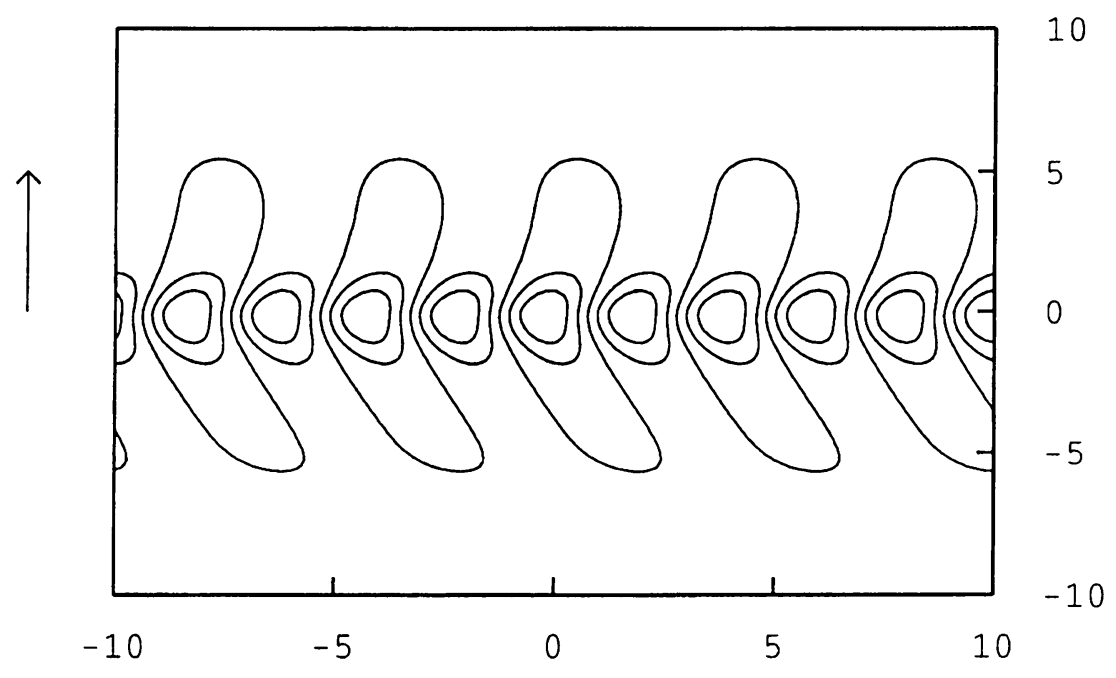

FIG. 5a. The streamlines of the perturbed velocity field for $R=10$, $k=1.55, Z=8, P=2.5, u=\sqrt{2}, \phi=7.9094$ 


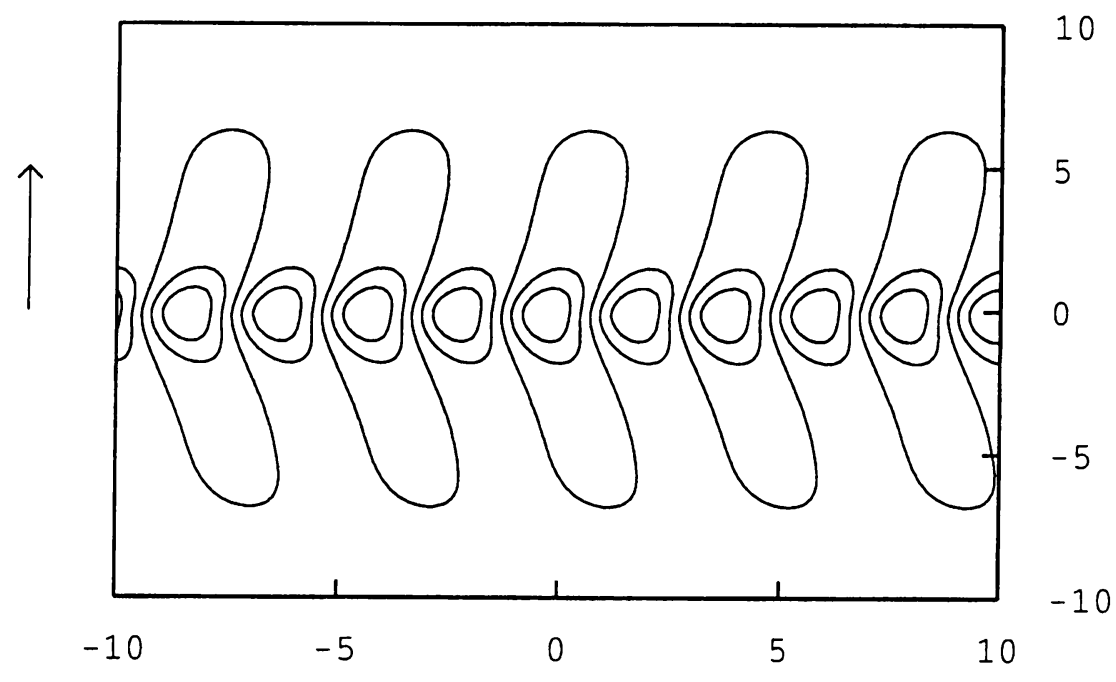

FIG. 5b. The streamlines of the perturbed velocity field for $R=10$, $k=1.55, Z=8, P=4.5, u=\sqrt{2}, \phi=7.9176$

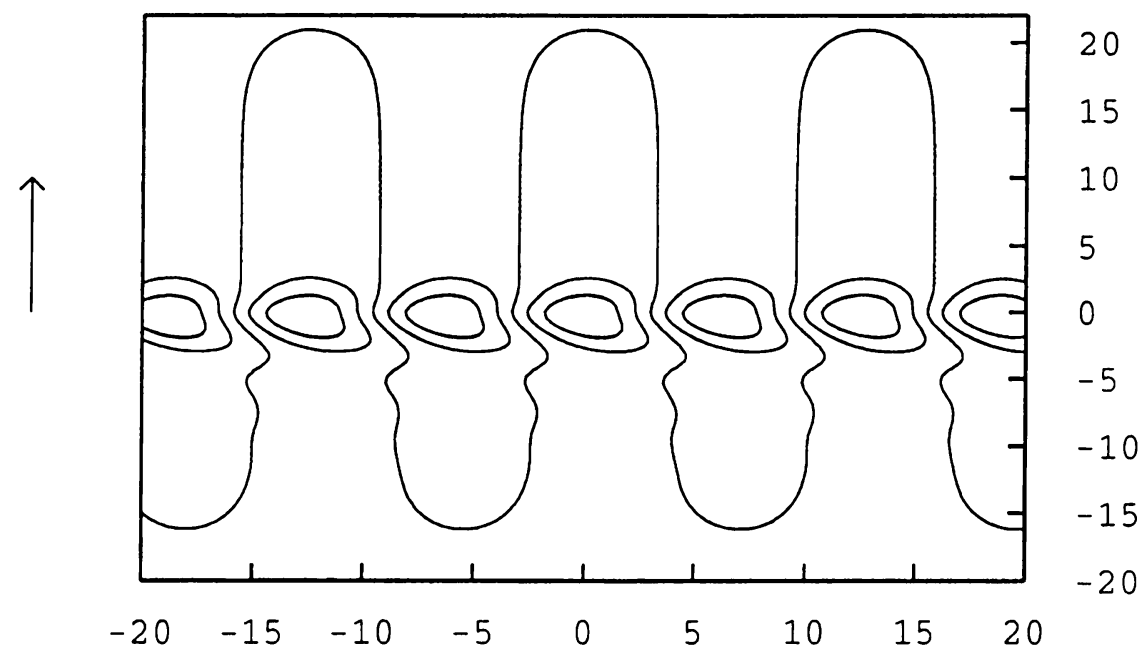

FIG. 5c. The streamlines of the perturbed velocity field for $R=20$, $k=0.5, Z=7.795, P=0.5, u=\sqrt{2}, \phi=3.945$

As in subsection 6.1, Figs. 11a, 12a, 13a (see pp. 27-29) show the streamlines of the perturbed velocity field for the ascending and descending fronts, for the cellular (steady) stability boundary in the parameter space. Figures 11b, 12b, 13b (see pp. 28-30) show the perturbation of the temperature and the component $\tilde{v}_{z}$ of the velocity as a function of $z$. Figures 14a and 14b (see pp. 30 and 31) show the same functions for the case of a solid product for the front propagating upward. 


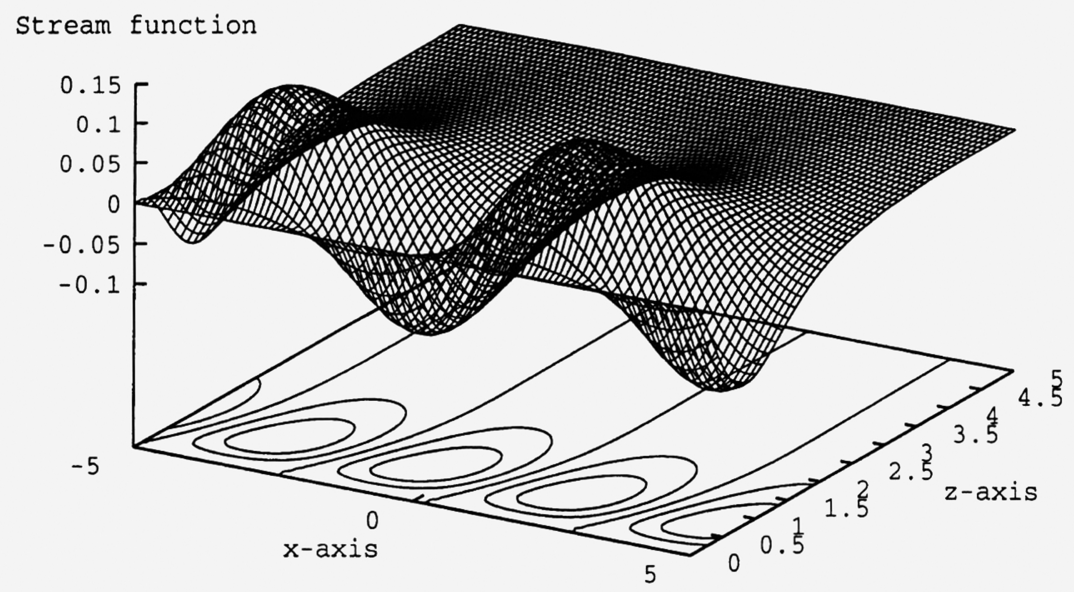

FIG. 6a. The streamlines of the perturbed velocity field (in the case of a solid product and an ascending front) for $R=40, k=1.2$, $Z=8, P=0.5, u=\sqrt{2}, \phi=4.536$

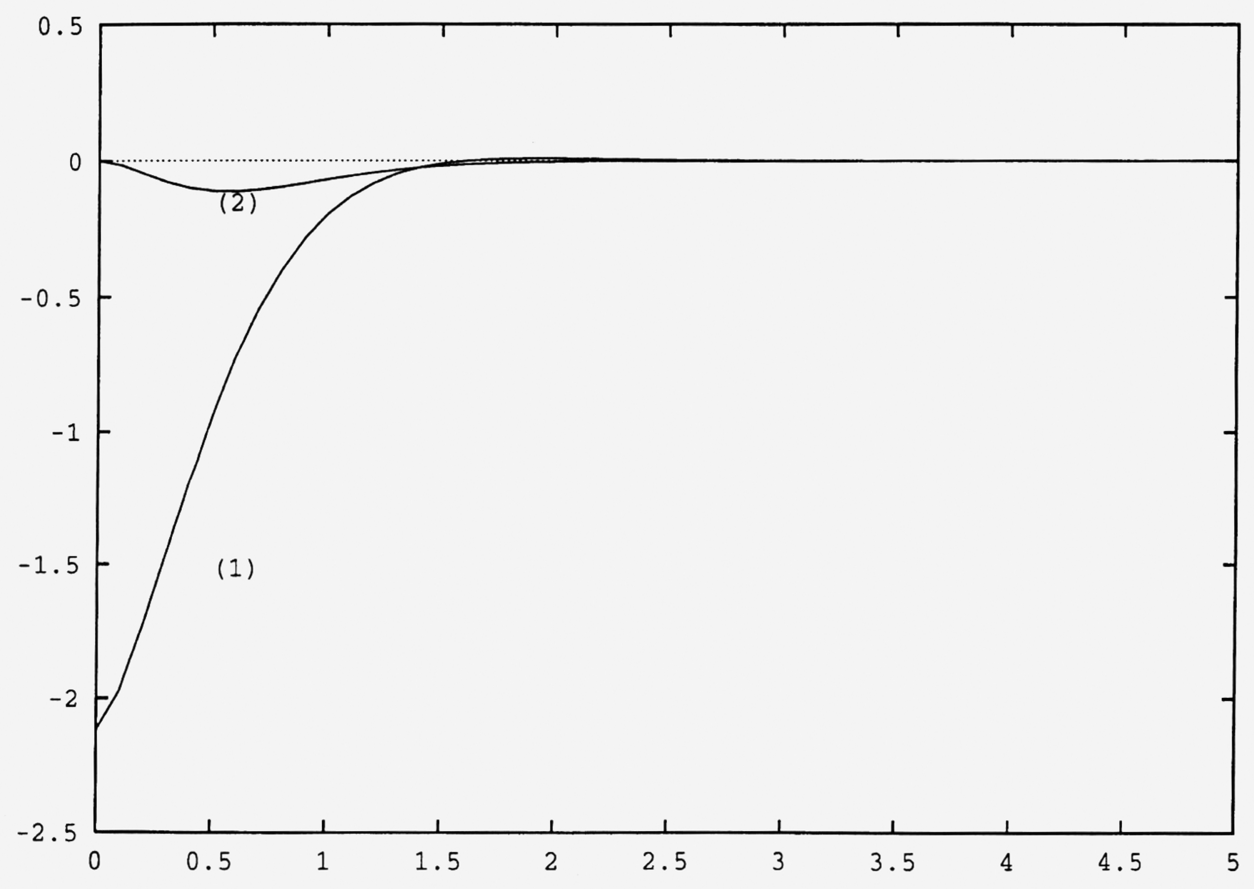

FIG. 6b. (1) $\tilde{\theta}$ as a function of the variable $z,(2) \tilde{v}_{z}$ as a function of $z$ for $R=40, k=1.2, Z=8, P=0.5, u=\sqrt{2}, \phi=4.536$

Figure 15 (see p. 32) qualitatively shows the stability boundary in the $(R, Z)$-plane for different values of the wavenumber. 


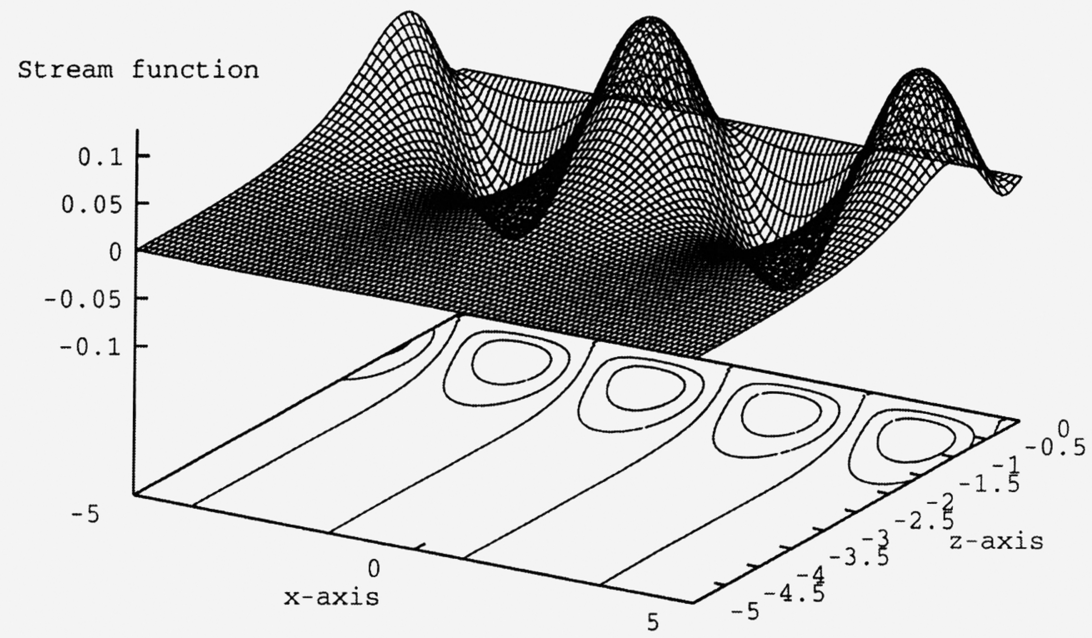

FIG. 7a. The streamlines of the perturbed velocity field (in the case of a solid product and a descending front) for $R=-20, k=1.3$, $Z=8, P=0.5, u=\sqrt{2}, \phi=4.833$

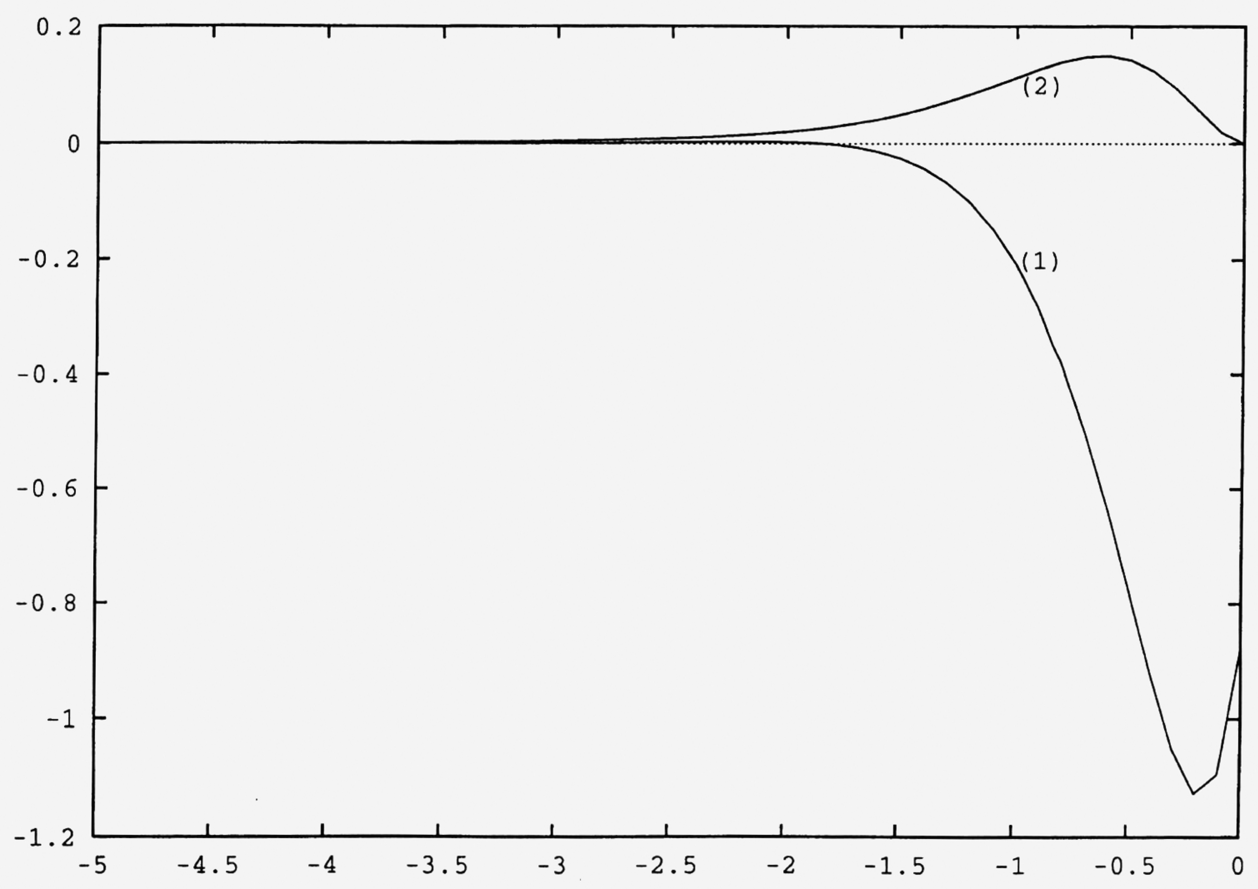

FIG. 7b. (1) $\tilde{\theta}$ as a function of the variable $z,(2) \tilde{v}_{z}$ as a function of $z$ for $R=-20, k=1.3, Z=8, P=0.5, u=\sqrt{2}, \phi=4.833$ 


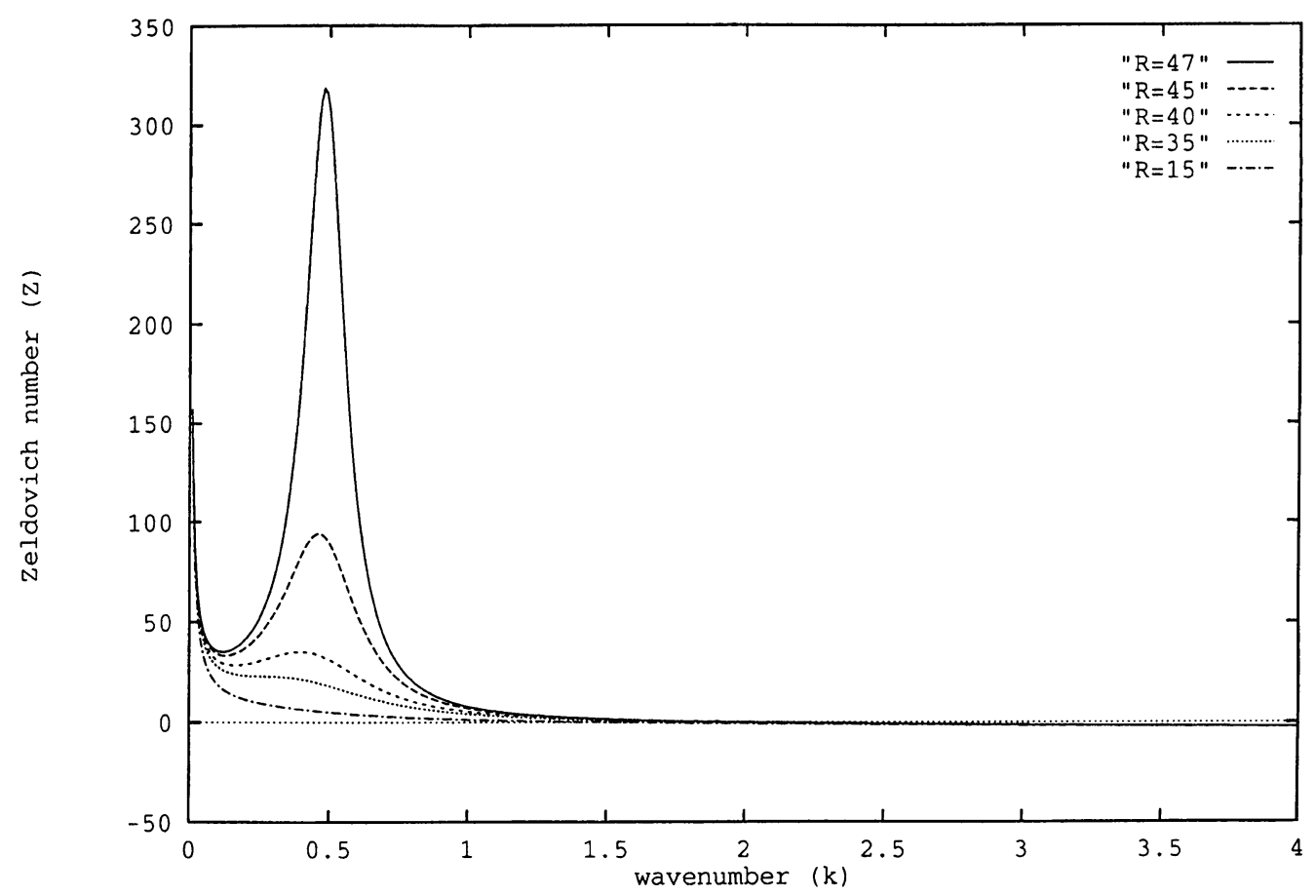

FIG. 8. Critical value of $Z$ as a function of $k$ for different values of $R ; P=0.5, u=\sqrt{2}$

\section{Discussion.}

7.1. The influence of the reaction on convective instability. We begin the discussion of the results obtained in the case of cellular instability.

We recall the main result in the case of a front with a solid product: ascending fronts are unstable for sufficiently large Rayleigh numbers while descending fronts are always stable. This is to some extent similar to the Rayleigh-Bénard convection for a layer of liquid heated from below or from above, and also has a clear physical interpretation. For the front propagating upward the hot products of the reaction are below the cold original reactants and heat them. It can cause convection of the liquid above the front. For a front propagating downward hot products of the reaction heat the cold liquid from above, and there is no reason for convection to occur.

The principal difference for the case of a liquid product is that convective instability can occur for a descending front also (Fig. 9 on p. 26). We give the following physical explanation of this phenomenon: Suppose there is a temperature perturbation on the front, i.e., a small spot where the temperature is greater than the adiabatic one. It can cause the liquid motion as shown in Fig. 13a (see p. 29). The liquid motion toward the hot spot decreases the heat loss from it due to heat diffusion and causes a further increase of temperature. This self-accelerating process can lead to instability. 


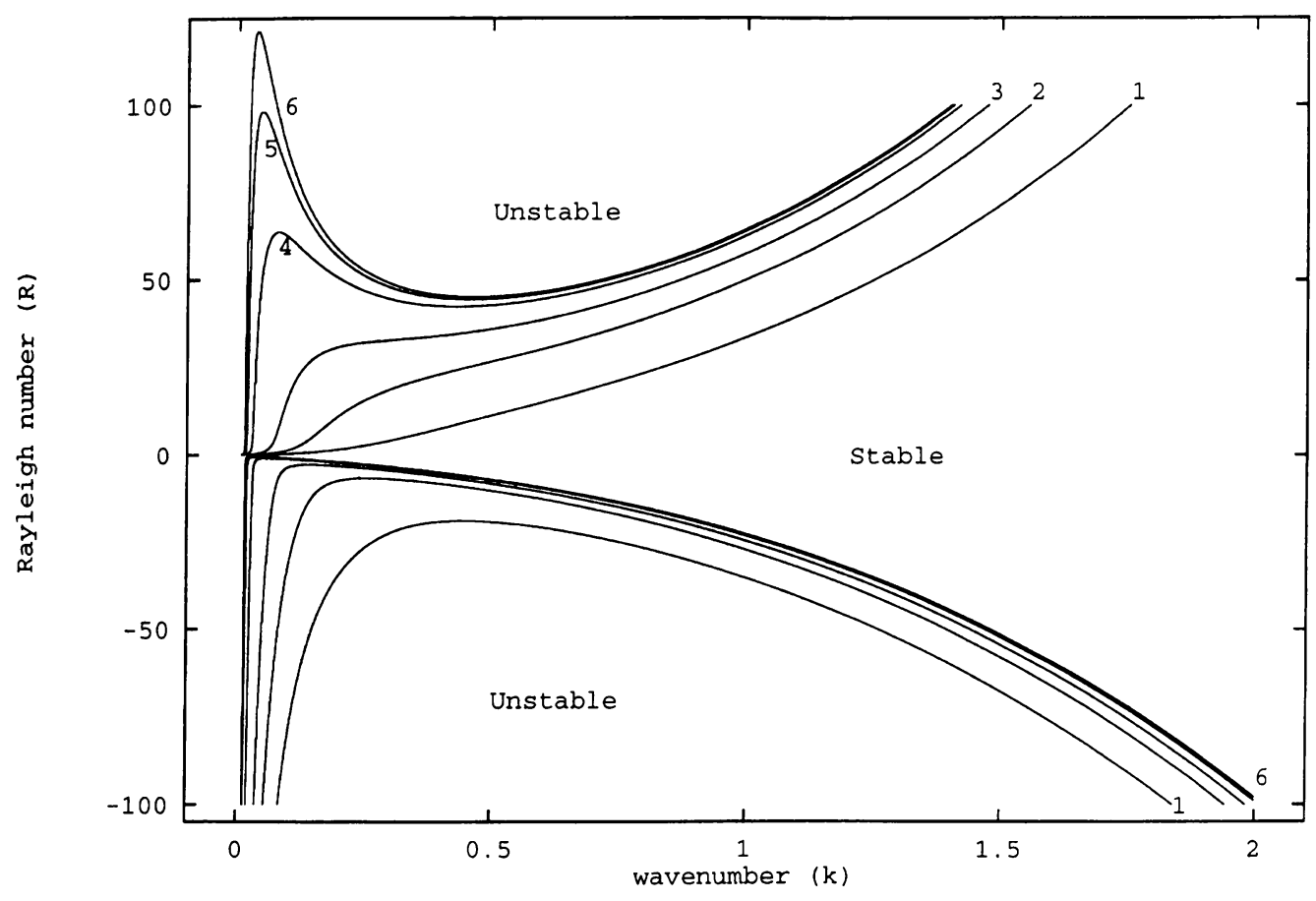

FIG. 9. Critical value of $R$ as a function of $k$ for different values of $Z ; P=0.5, u=\sqrt{2}$, (1) $Z=8$, (2) $Z=20$, (3) $Z=40$, (4) $Z=100$, (5) $Z=160$, (6) $Z=400$

The asymptotic results show that descending fronts become less stable with increase of the Zeldovich number (Fig. 9). We recall that this parameter determines the temperature dependence of the reaction rate. The greater the Zeldovich number, the less the temperature interval where the reaction occurs. Thus if $Z$ is large, in the framework of the mechanisms described before, the liquid comes to the hot spot unreacted and the whole temperature release due to the reaction occurs there. If $Z$ is small, the liquid comes there partially reacted, and the increase of temperature at the hot spot is less.

It is interesting that for ascending fronts the effect of increase of $Z$ is opposite. For large Zeldovich numbers the front is more stable (Fig. 9). It can also be explained by means of the temperature dependence of the reaction rate. Consider a small drop of liquid that moves from the front upward because of convection (Fig. 11a). Two processes should be taken into account: heat release because of the reaction and heat loss because of diffusion from the drop to the surrounding medium. When the drop goes away from the front, the temperature of the surrounding medium decreases and the temperature in the drop also decreases. In the case of large $Z$, the reaction in the drop stops, the drop cools and the driving force of convection disappears. For small $Z$, the reaction can continue even if the drop is rather far from the front. It keeps the temperature in the drop higher and supports convection.

We note that for a solid product the stability condition does not depend on $Z$ (see [6]). Probably it can be explained by the location of the vortices with respect to the 


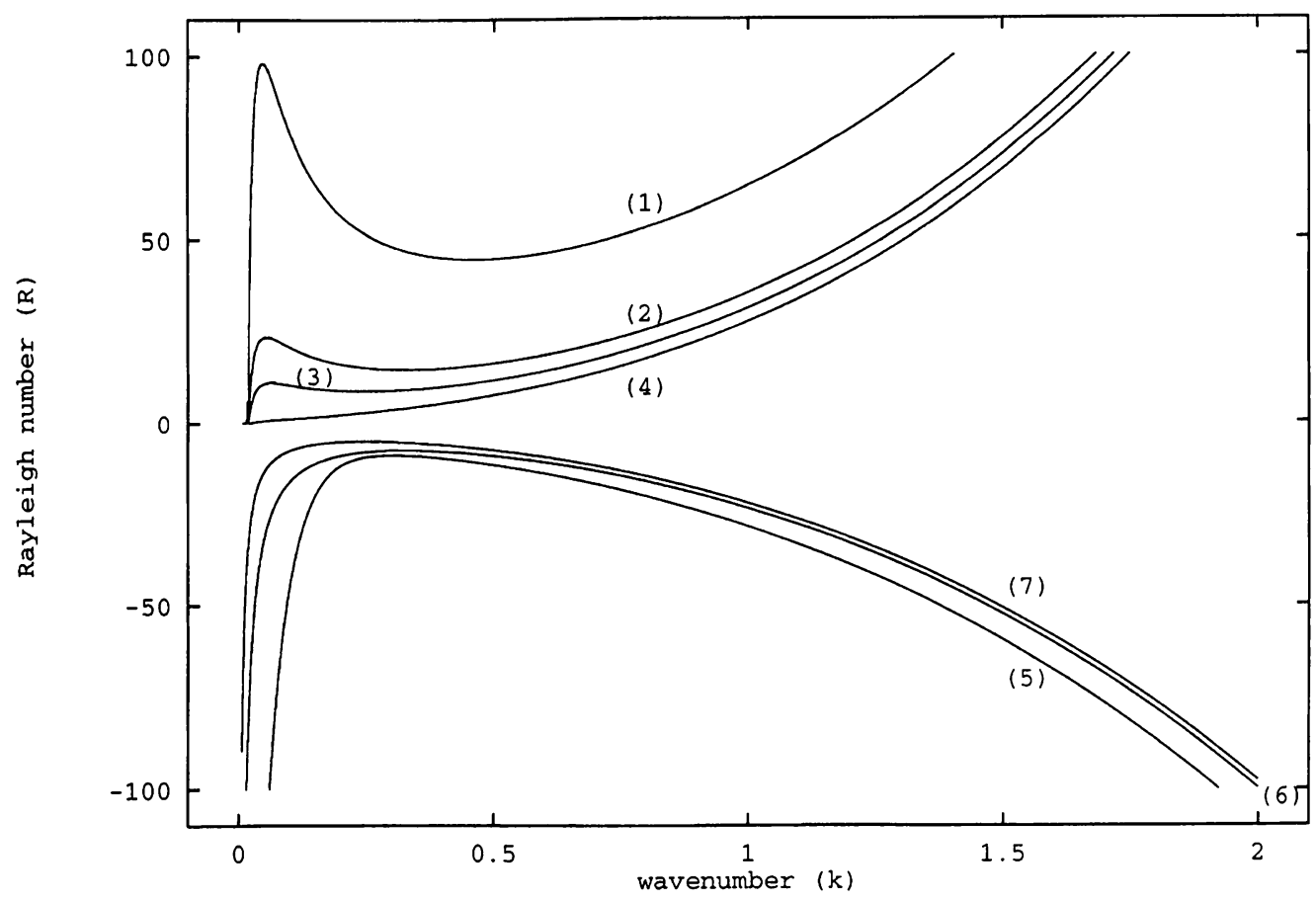

FIG. 10. Critical value of $R$ as a function of $k$ for different values of $P$; $u=\sqrt{2}, Z=160$, (1) $P=0.5$, (2) $P=1.5$, (3) $P=2.5$, (4) $P=10.5, Z=16$, (5) $P=0.5$, (6) $P=1.5$, (7) $P=2.5$

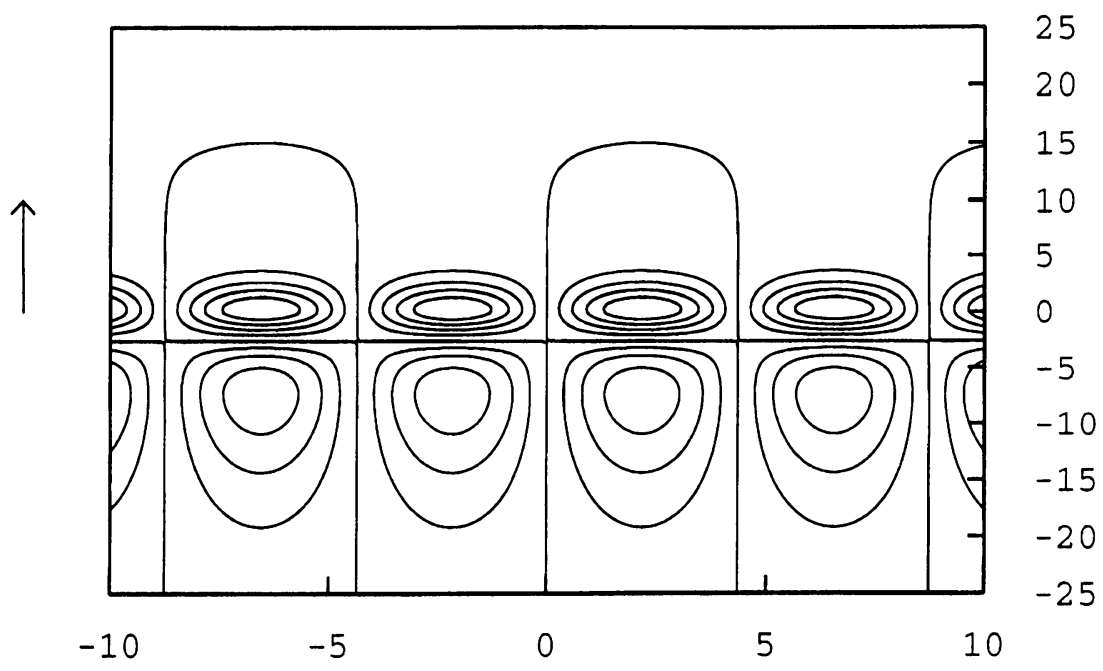

FIG. 11a. The streamlines of the perturbed velocity field (ascending front) for $R=20, k=0.7195, Z=8, P=0.5, u=\sqrt{2}, \omega=0$ 


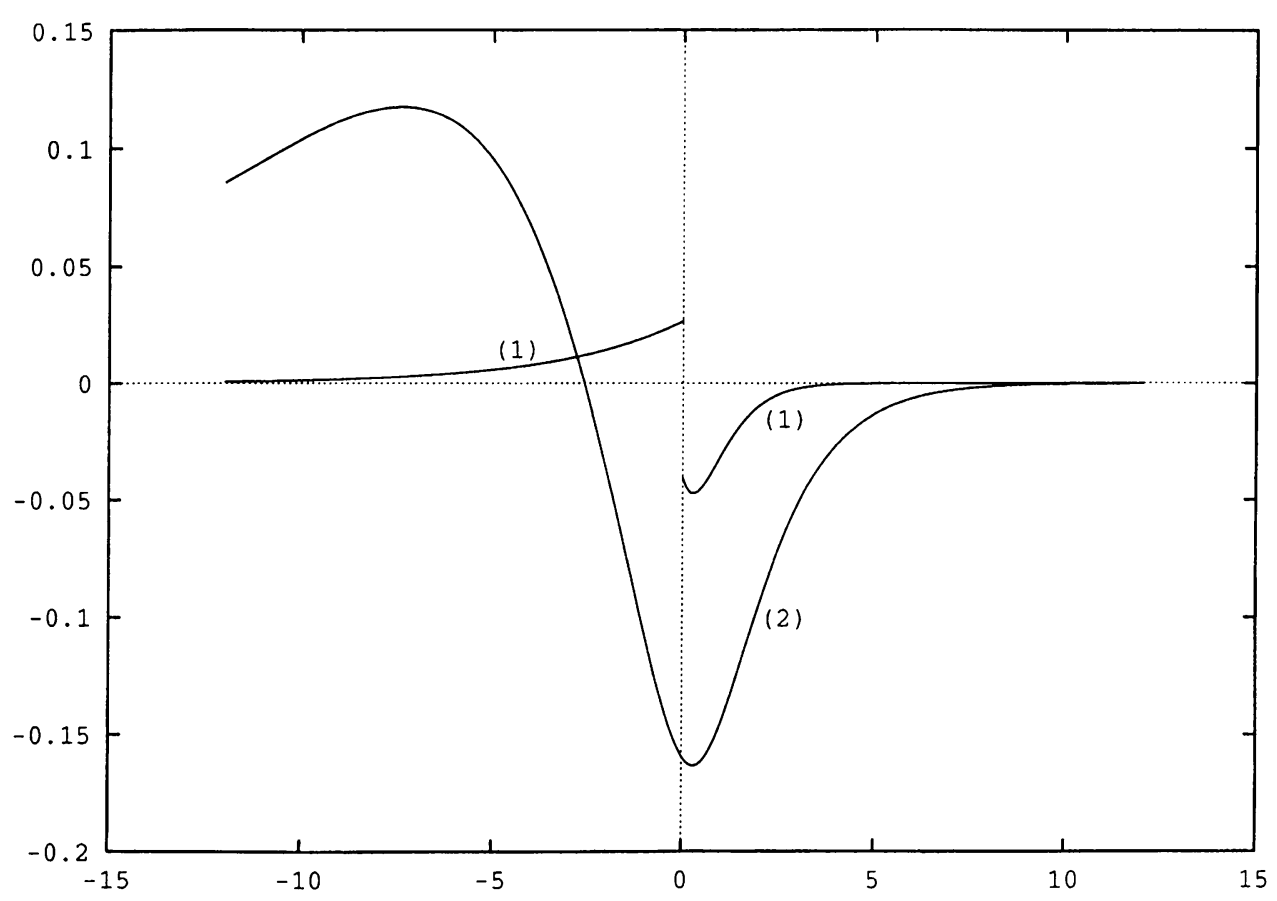

Fig. 11b. (1) $\tilde{\theta}$ as a function of the variable $z,(2) \tilde{v}_{z}$ as a function of $z$ for $R=20, k=0.7195, Z=8, P=0.5, u=\sqrt{2}, \omega=0$

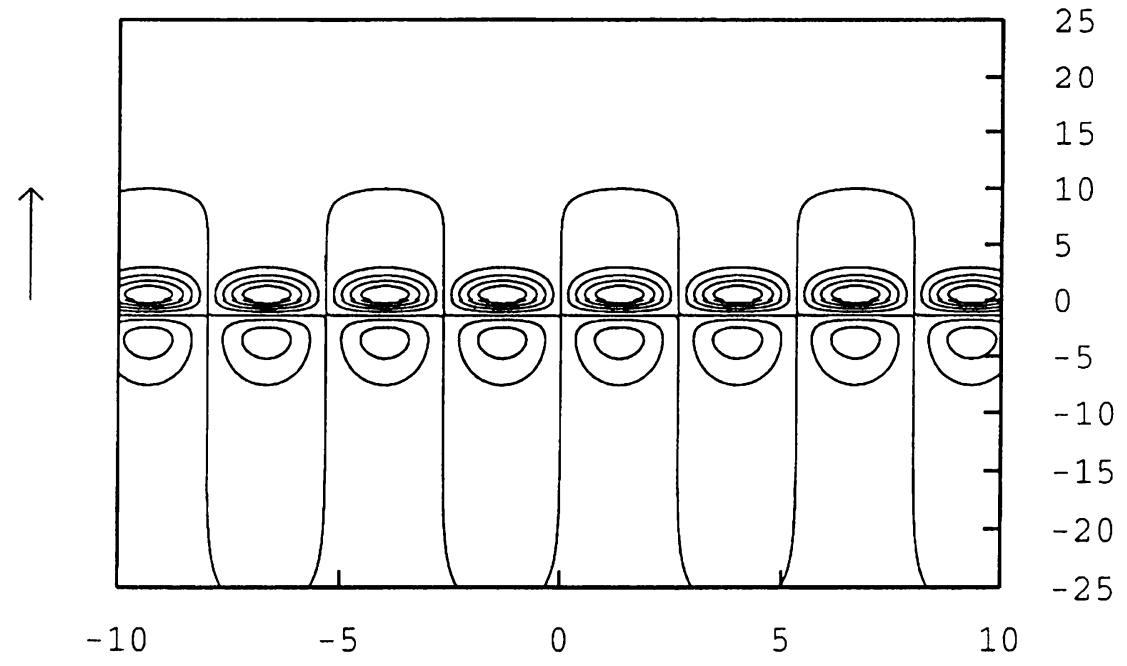

FIG. 12a. The streamlines of the perturbed velocity field (ascending front) for $R=45, k=1.785, Z=8, P=0.5, u=\sqrt{2}, \omega=0$

front. In the case of a solid product the velocity component $v_{x}$ decreases the heat loss from the drop while in the case of a liquid product this effect is less essential. 


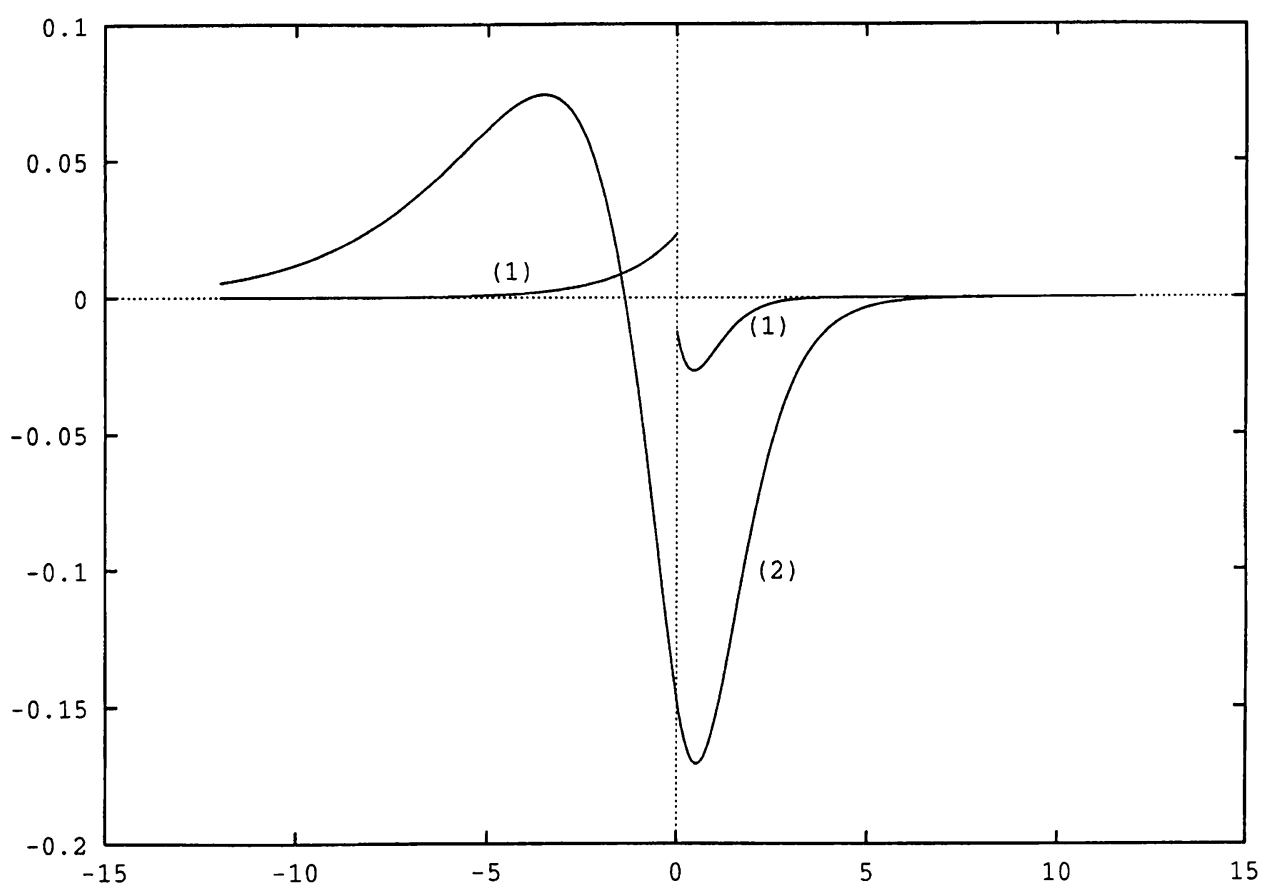

FIG. 12b. (1) $\tilde{\theta}$ as a function of the variable $z,(2) \tilde{v}_{z}$ as a function of $z$ for $R=45, k=1.785, Z=8, P=0.5, u=\sqrt{2}, \omega=0$

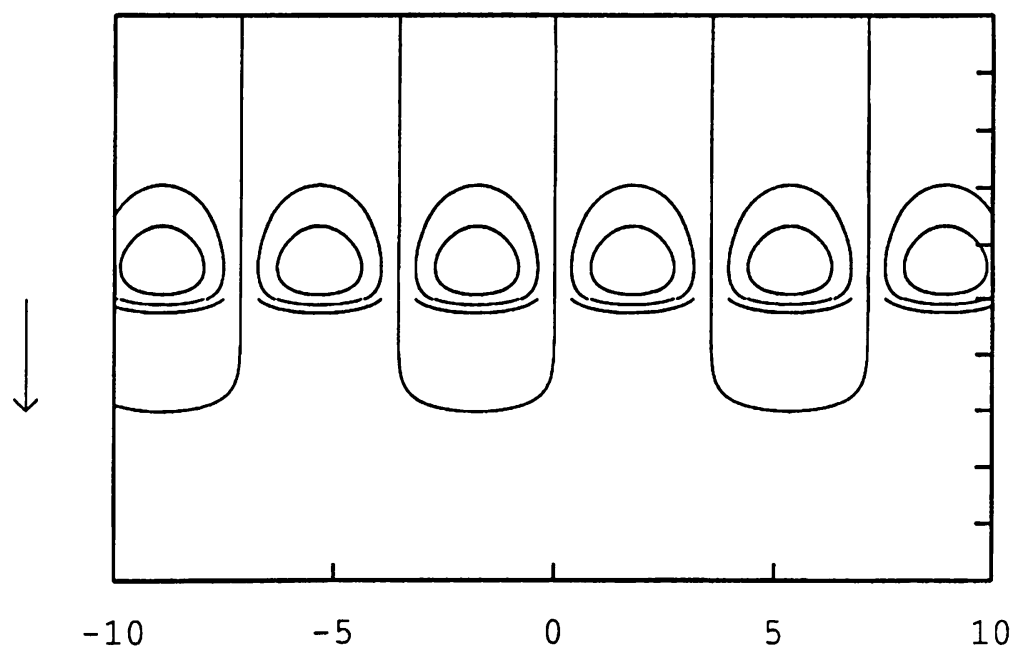

FIG. 13a. The streamlines of the perturbed velocity field (descending front) for $R=-30, k=0.8805, Z=8, P=0.5, u=\sqrt{2}, \omega=0$

One more difference between the cases of solid and liquid products is that for the first the temperature perturbation behind the front is always zero [6], while for the second this is not so. As before this can be explained in terms of interaction between heat diffusion 


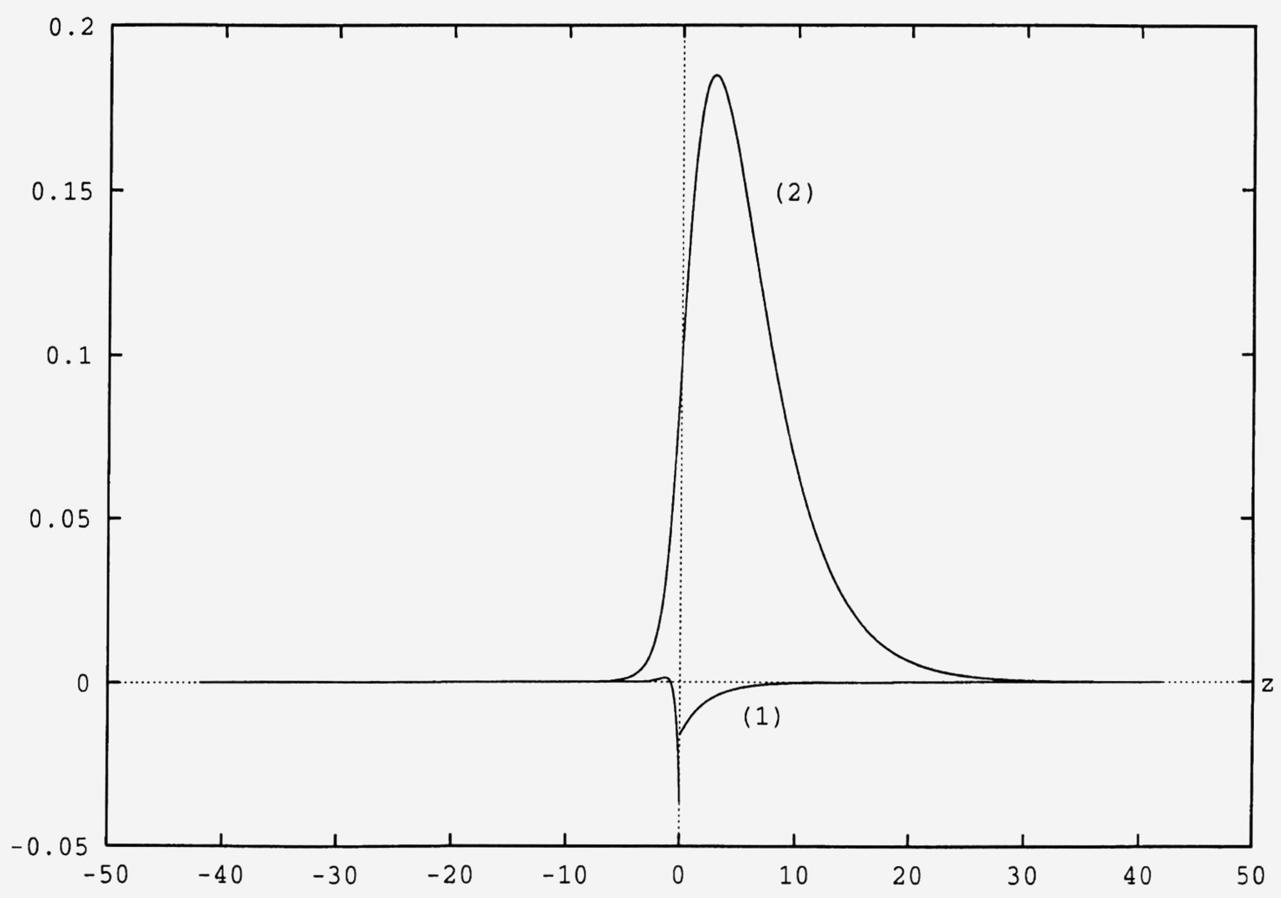

FIG. 13b. (1) $\tilde{\theta}$ as a function of the variable $z,(2) \tilde{v}_{z}$ as a function of $z$ for $R=-30, k=0.8805, Z=8, P=0.5, u=\sqrt{2}, \omega=0$

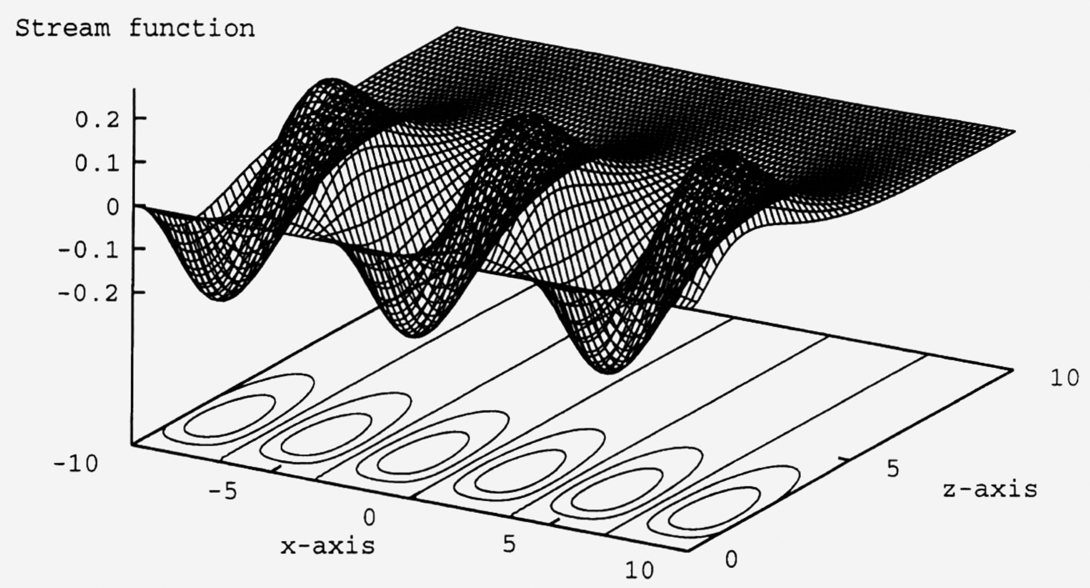

FIG. 14a. The streamlines of the perturbed velocity field (in the case of a solid product and an ascending front) for $R=35, k=0.9015$, $P=2, u=\sqrt{2}, \omega=0$

and hydrodynamics. The temperature perturbation before the front induces that behind it. In the case of a solid product the temperature difference behind the front is decreased by heat diffusion. In the case of a liquid product there is also liquid motion behind the front that decreases the effect of diffusion. 


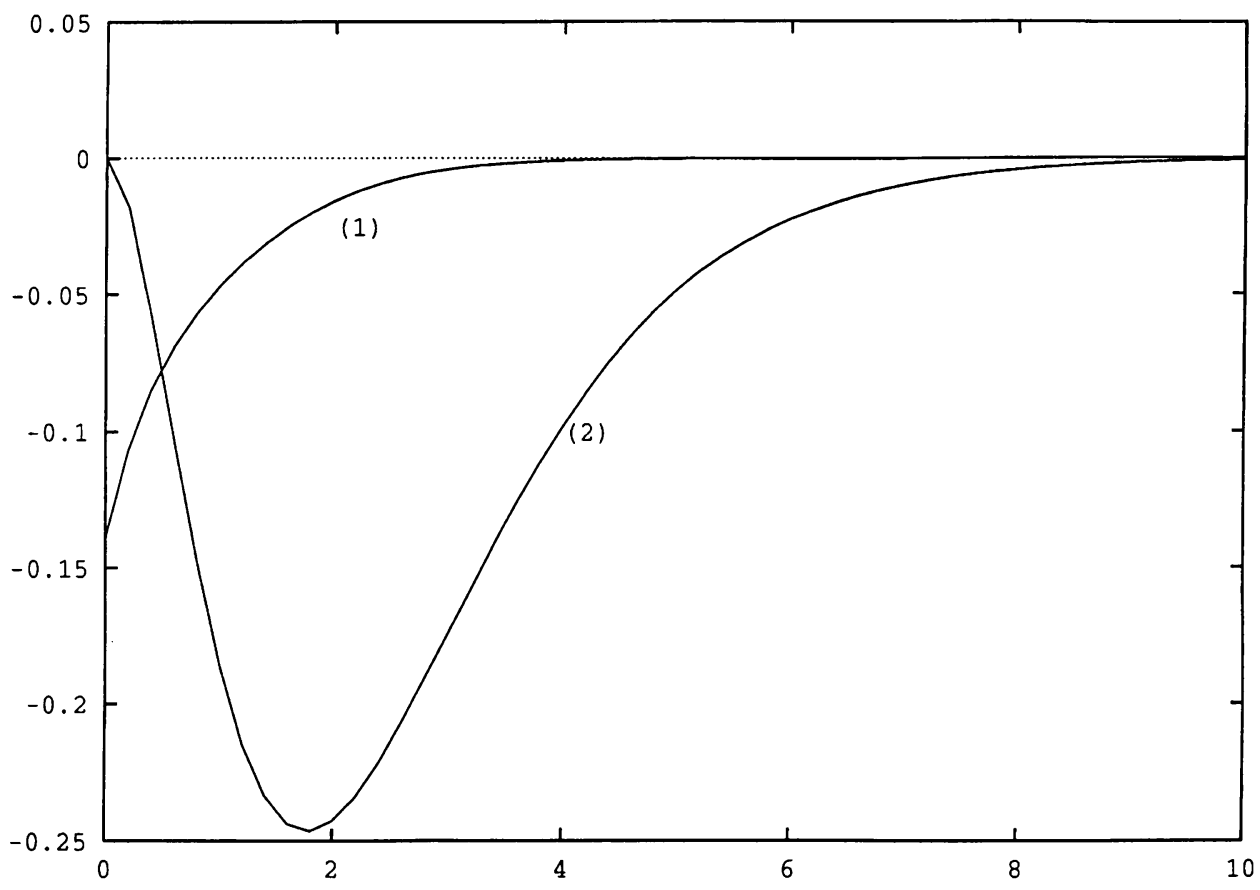

FIG. 14b. (1) $\tilde{\theta}$ as a function of the variable $z,(2) \tilde{v}_{z}$ as a function of $z$ for $R=35, k=0.9015, P=2, u=\sqrt{2}, \omega=0$

Two remarks about convective instability concern the number of vortices and the long-wave instability.

In the case of an ascending front and a solid product there is only one vortex ahead of the front (Fig. 14a). For the case of a liquid product there is one more vortex behind it (Fig. 11a, for example) induced by the first one. The liquid motion is not caused by gravity but is opposite it. This means that a part of the liquid with a higher temperature goes down while more cold liquid goes up. In the case of a descending front there is only one vortex behind the front (Fig. 13a).

Finally, consider the behaviour of the stability boundary as $k \rightarrow 0$. For an ascending front and a solid product the critical value of the Rayleigh number tends to a positive constant as $k \rightarrow 0$ [6]; in the case of a liquid product it tends to zero (Fig. 9). Thus a solid product stabilizes the front.

7.2. The influence of convection on thermal instability. We now proceed to discuss oscillatory instability. We again recall the main result for the case of a solid product. Ascending fronts $(R>0)$ are more stable than those without hydrodynamics $(R=0)$; descending fronts $(R<0)$ are less stable [6]. The explanation of the influence of convection on oscillatory instability is quite clear: for the front propagating upward, convective motion increases the heat loss from the front; for the front propagating downward, it is decreased.

It appears that in the case of a liquid product the influence of hydrodynamics is opposite. In the case of ascending fronts $(R>0)$ the front is less stable than for $R=0$ 


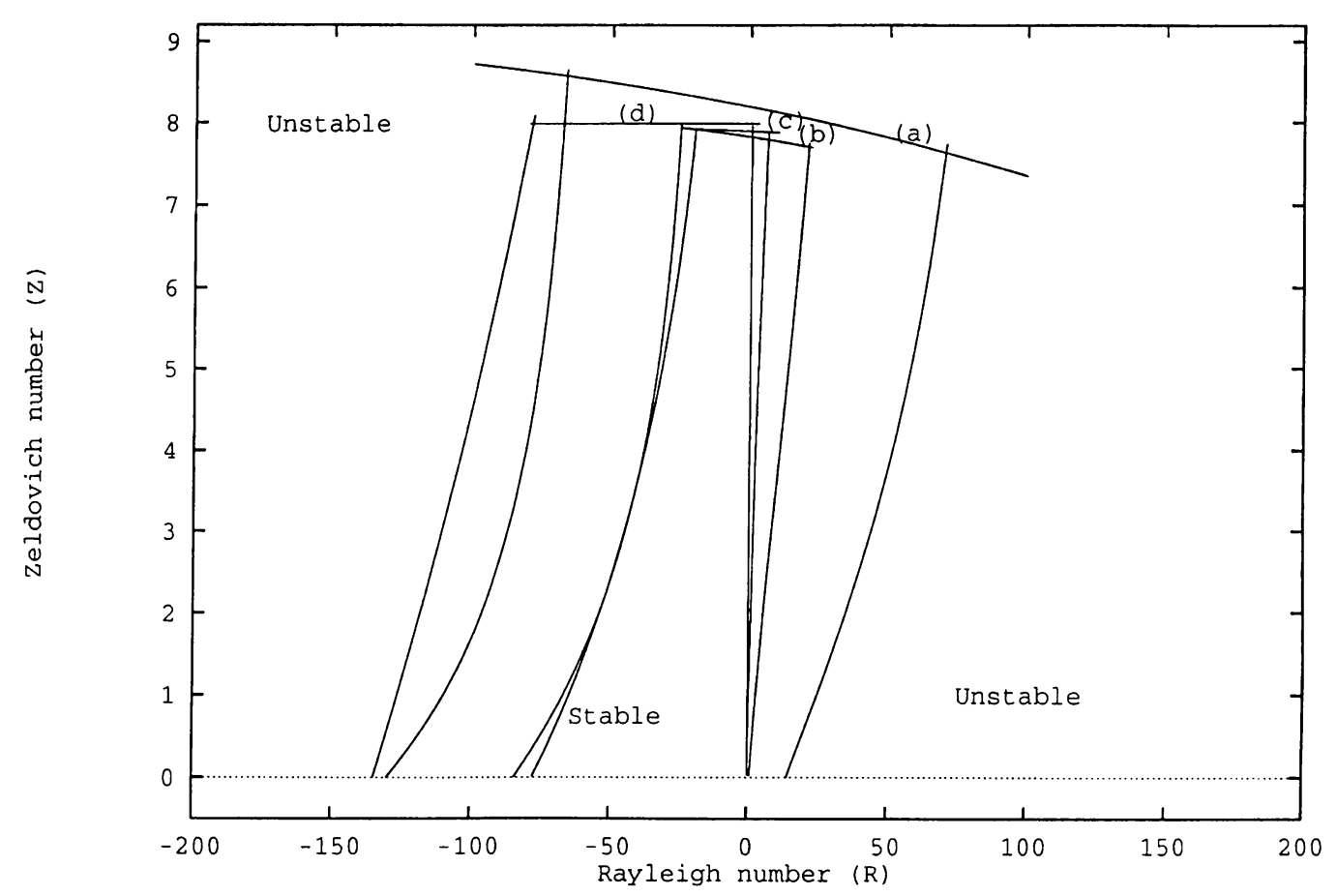

FIG. 15. Stability domain on $(R, Z)$-plane for different values of $k$; $P=0.5, u=\sqrt{2}$, (a) $k=1.5$, (b) $k=0.75$, (c) $k=0.3705$, (d) $k=0.1$

(without hydrodynamics); in the case of descending fronts $(R<0)$ the front is more stable (Fig. 1). To give a physical explanation of this effect we should take into account the following factors which determine the front stability in the case of ascending fronts $(R>0)$ :

(1) Convection increases the front's perturbations. Indeed, if there is a small perturbation of the front, then the absolute value of the temperature gradient before the front becomes greater. Thus it can cause a convection motion that makes the perturbation larger. In its turn, it increases the gradient, etc.

(2) Convection increases the heat loss from the front. This effect is more essential for the case of a solid product rather than for a liquid product. In the first case the convective motion mixes the liquid only ahead of the front; in the second case, it mixes the hot product also. So the temperature at the front is greater.

(3) In the case of a solid product the convective motion prevents the propagation of a perturbation along the front since the motion is towards the perturbation (see Figs. $6 \mathrm{a}, 7 \mathrm{a})$. In the case of a liquid product the liquid motion does not directly influence the propagation of the perturbation.

Thus for a solid product (1) destabilizes the front, whereas (2) and (3) stabilize it and finally make it more stable. For a liquid product (1) destabilizes the front, (2) stabilizes 
it to a less extent than for a solid product, while (3) does not influence it. The front is less stable.

The case of descending fronts can be considered similarly.

One more remark concerns velocity fields. Figures $2 \mathrm{a}, 3 \mathrm{a}, 4 \mathrm{a}, 5 \mathrm{a}, 5 \mathrm{~b}, 5 \mathrm{c}$ show that they can be different and rather complicated. In some cases (Fig. 5c), the level curves of the stream function go from $-\infty$ to $+\infty$ in the $x$ variable. It is a consequence of the formulation of the model problem (3.1)-(3.4) in a moving coordinate system attached to the front position, but this does not mean that there are some particles of the liquid that move from $-\infty$ to $+\infty$. Rather the whole structure moves and the motion of the liquid is up and down about some average position.

We are currently doing the numerical computation of the complete problem (2.1)(2.4), using an adaptive pseudo-spectral domain decomposition [4]. The first numerical results seem to be in agreement with the analytical results described in this paper.

8. Appendix. In this Appendix we describe the method used to derive the matching conditions (3.17)-(3.22). We consider both the outer and the inner expansions of the velocity and recall that $\eta=z_{1} \varepsilon^{-1}$ where $\varepsilon$ is a small parameter. We have

$$
\begin{aligned}
& v\left(x, y, z_{1}\right)=v_{0}\left(x, y, z_{1}\right)+\varepsilon v_{1}\left(x, y, z_{1}\right)+\varepsilon^{2} v_{2}\left(x, y, z_{1}\right)+\varepsilon^{3} v_{3}\left(x, y, z_{1}\right)+O\left(\varepsilon^{4}\right), \\
& v(x, y, \varepsilon \eta)=\tilde{v}_{0}(x, y, \eta)+\varepsilon \tilde{v}_{1}(x, y, \eta)+\varepsilon^{2} \tilde{v}_{2}(x, y, \eta)+\varepsilon^{3} \tilde{v}_{3}(x, y, \eta)+O\left(\varepsilon^{4}\right) .
\end{aligned}
$$

In determining the outer and the inner expansions, we use two different limit processes [17]: an outer limit defined by

$$
v_{0}\left(x, y, z_{1}\right)=\lim _{\substack{z_{1} \text { fixed } \\ \varepsilon \rightarrow 0}} v\left(x, y, z_{1}\right)
$$

and an inner limit defined by

$$
\tilde{v}_{0}(x, y, \eta)=\lim _{\substack{\eta \text { fixed } \\ \varepsilon \rightarrow 0}} v(x, y, \varepsilon \eta)
$$

To get (3.17) we use the matching principle:

$$
\lim _{z_{1} \rightarrow \pm 0} v_{0}\left(x, y, z_{1}\right)=\lim _{\eta \rightarrow \pm \infty} \tilde{v}_{0}(x, y, \eta)
$$

We write the outer expansion in terms of the inner variable $\eta$ and use the Taylor expansion:

$$
\begin{aligned}
v\left(x, y, z_{1}\right)= & v_{0}(x, y, 0)+\varepsilon\left(v_{0}^{\prime}(x, y, 0) \eta+v_{1}(x, y, 0)\right) \\
& +\varepsilon^{2}\left(\frac{1}{2} v_{0}^{\prime \prime}(x, y, 0) \eta^{2}+v_{1}^{\prime}(x, y, 0) \eta+v_{2}(x, y, 0)\right) \\
& +\varepsilon^{3}\left(\frac{1}{6} v_{0}^{\prime \prime \prime}(x, y, 0) \eta^{3}+\frac{1}{2} v_{1}^{\prime \prime}(x, y, 0) \eta^{2}+v_{2}^{\prime}(x, y, 0) \eta+v_{3}(x, y, 0)\right) \\
& +O\left(\varepsilon^{4}\right) .
\end{aligned}
$$


Using the outer limit and the inner limit process again we have

$$
\begin{gathered}
v_{0}^{\prime}(x, y, 0) \eta+v_{1}(x, y, 0)=\lim _{\substack{z_{1} \text { fixed } \\
\varepsilon \rightarrow 0}} \frac{v\left(x, y, z_{1}\right)-v_{0}(x, y, 0)}{\varepsilon}, \\
\tilde{v}_{1}(x, y, \eta)=\lim _{\substack{\eta \text { fixed } \\
\varepsilon \rightarrow 0}} \frac{v(x, y, \varepsilon \eta)-\tilde{v}_{0}(x, y, \eta)}{\varepsilon} .
\end{gathered}
$$

The matching principle gives (3.18). We repeat this process to give the other matching conditions.

\section{REFERENCES}

[1] A. P. Aldushin and S. G. Kasparyan, Thermodiffusional instability of a combustion front, Soviet Physics-Doklady, Akademii Nauk SSSR (5) 24, 29 (1979)

[2] G. I. Barenblatt, Ya. B. Zeldovich, and A. G. Istratov, Diffusive-thermal stability of a laminar flame, Zh. Prikl. Mekh. Tekh. Fiz. (4) 21 (1962) (in Russian)

[3] P. Clavin, Dynamic behavior of premixed flame fronts in laminar and turbulent flows, Progr. Energy Comb. Sci. 11, 1-59 (1985)

[4] F. Desprez and M. Garbey, Numerical simulation of a combustion problem on a Paragon Machine, to appear in Parallel Computing

[5] D. A. Frank-Kamenetskii, Diffusion and Heat Transfer in Chemical Kinetics, Plenum Press, New York, 1969

[6] M. Garbey, A. Taïk, and V. Volpert, Linear stability analysis of reaction fronts in liquids, Quart. Appl. Math. 54, 225-247 (1996)

[7] A. G. Istratov and V. B. Librovich, Effect of the transfer processes on stability of a planar flame front, J. Appl. Math. Mech. (3) 30, 451-466 (1966) (in Russian)

[8] L. D. Landau and E. M. Lifshitz, Fluid Mechanics, Pergamon, Oxford and New York, 1987

[9] O. Manley and M. Marion, Attractor dimension for a simple premixed flame propagation model, Combustion Sci. and Tech. 88, 1532 (1992)

[10] M. Marion, Attractors and Turbulence for some Combustion Models, Mathematics and its Applications, vol. 35 , to appear

[11] S. B. Margolis, H. G. Kaper, G. K. Leaf, and B. J. Matkowsky, Bifurcation of pulsating and spinning reaction fronts in condensed two-phase combustion, Combustion Sci. and Tech. 43, 127-165 (1985)

[12] S. B. Margolis, An asymptotic theory of condensed two-phase flame propagation, SIAM J. Appl. Math. 43, 351-369 (1983)

[13] M. Matalon and B. J. Matkowsky, Flames in fluids: Their interaction and stability, Combustion Science and Tech. 34, 295-316 (1983)

[14] B. J. Matkowsky and G. I. Sivashinsky, Propagation of a pulsating reaction front in solid fuel combustion, SIAM J. Appl. Math. 35, 465-478 (1978)

[15] B. J. Matkowsky and G. I. Sivashinsky, Acceleration effects on the stability of flame propagation, SIAM J. Appl. Math. 37, 669-685 (1979)

[16] B. J. Matkowsky and G. I. Sivashinsky, An asymptotic derivation of two models in flame theory associated with the constant density approximation, SIAM J. Appl. Math. 37, 686-699 (1979)

[17] A. H. Nayfeh, Perturbation Methods, Wiley, New York, 1973

[18] B. V. Novozhilov, The rate of propagation of the front of an exothermic reaction in a condensed phase, Proc. Academy Sci. USSR, Phys. Chem. Sect. 141, 836 838 (1961)

[19] J. A. Pojman, R. Graven, A. Khan, and W. West, Convective instabilities in traveling fronts of addition polymerization, J. Physical Chemistry 96, 7466-7472 (1992)

[20] J. A. Pojman and I. R. Epstein, Convective effects on chemical waves. 1. Mechanisms and stability criteria, J. Phys. Chem. (12) 94, 4966-4972 (1990)

[21] J. A. Pojman, I. R. Epstein, T. J. McManus, and K. Scowalter, Convective effects on chemical waves. 2. Simple convection in the iodate-arsenous acid system, J. Phys. Chem. (3) 95, 1299-1306 (1991)

[22] K. G. Shkadinsky, B. I. Khaikin, and A. G. Merzhanov, Propagation of a pulsating exothermic reaction front in condensed phase, Combustion, Explosion, and Shock Waves 7, 15 (1971) 
[23] D. A. Vasquez, J. W. Wilder, and B. F. Edwards, Hydrodynamic instability of chemical waves, J. Chem. Phys. (3) 98, 2138-2143 (1993)

[24] D. A. Vasquez, B. F. Edwards, and J. W. Wilder, Onset of convection for autocatalytic reaction fronts: Laterally bounded systems, Physical Review A 43, 6694-6699 (1991)

[25] H. J. Viljoen, J. E. Gatica, and V. Hlavacek, Bifurcation analysis of chemically driven convection, Chem. Eng. Sci. (2) 45, 503-517 (1990)

[26] Vit. A. Volpert, Vl. A. Volpert, and J. A. Pojman, Effect of thermal expansion on stability of reaction front propagation, Chem. Eng. Sci. 49, No. 14, 2385-2388 (1994)

[27] J. W. Wilder, B. F. Edwards, D. A. Vasquez, and G. I. Sivashinksy, Derivation of a nonlinear front evolution equation for chemical waves involving convection, Physica D, 73, 217-226 (1994)

[28] Ya. B. Zeldovich, G. I. Barenblatt, V. B. Librovich, and G. M. Makhviladze, The Mathematical Theory of Combustion and Explosions, Consultants Bureau, New York, 1985

[29] Ya. B. Zeldovich and D. A. Frank-Kamenetsky, Theory of thermal propagation of flames, Zh. Fiz. Khim. 12, 100 (1938) 